\title{
Precoded FIR and Redundant V-BLAST Systems for Frequency-Selective MIMO Channels
}

\author{
Chun-yang Chen, Student Member, IEEE, and P. P. Vaidyanathan, Fellow, IEEE
}

\begin{abstract}
The vertical Bell labs layered space-time (V-BLAST) system is a multi-input multioutput (MIMO) system designed to achieve good multiplexing gain. In recent literature, a precoder, which exploits channel information, has been added in the V-BLAST transmitter. This precoder forces each symbol stream to have an identical mean square error (MSE). It can be viewed as an alternative to the bit-loading method. In this paper, this precoded V-BLAST system is extended to the case of frequency-selective MIMO channels. Both the FIR and redundant types of transceivers, which use cyclic-prefixing and zero-padding, are considered. A fast algorithm for computing a cyclic-prefixing-based precoded V-BLAST transceiver is developed. Experiments show that the proposed methods with redundancy have better performance than the SVD-based system with optimal powerloading and bit loading for frequency-selective MIMO channels. The gain comes from the fact that the MSE-equalizing precoder has better bit-error rate performance than the optimal bitloading method.
\end{abstract}

Index Terms-Bit loading, decision feedback equalizer (DFE), frequency-selective multi-input multioutput (MIMO) channels, intersymbol-interference (ISI) MIMO channels, MIMO orthogonal frequency-division multiplexing (OFDM), precoder, vertical Bell labs layered space-time (V-BLAST).

\section{INTRODUCTION}

$\mathbf{I}$ N AN MIMO communication system, multiple transmission paths can be used to improve diversity and/or multiplexing gain [17]. The vertical Bell labs layered space-time (V-BLAST) system suggested in [10] is one of the MIMO transceivers systems designed to achieve good multiplexing gain. In the V-BLAST transmitter, every antenna transmits its own independently coded symbol. The V-BLAST receiver is a spatial-domain decision feedback equalizer (DFE) as shown in Fig. 1. In this scheme, one by one, the symbols are decoded by a linear minimum mean square error (LMMSE) estimator $\mathbf{f}_{k}$ followed by a slicer. Then, the decoded symbol is fed back to cancel its interference with other symbols. This process repeats until all of the symbols are decoded. The decoding order can be optimized by decoding the symbol with the largest signal-to-noise ratio (SNR) first to reduce the error propagation. Due to this decision feedback structure, the V-BLAST system has very good spectral efficiency in a scattering rich environment [10].

Manuscrfipt received January 19, 2006; revised October 19, 2006. This work was supported in part by the National Science Foundation under Grant CCF0428326, in part by ONR Grant N00014-06-1-0011, and in part by the California Institute of Technology. The associate editor coordinating the review of this manuscript and approving it for publication was Dr. Martin Haardt.

The authors are with the California Institute of Technology (Caltech), Pasadena, CA 91125 USA (e-mail: cyc@ caltech.edu).

Digital Object Identifier 10.1109/TSP.2007.894400

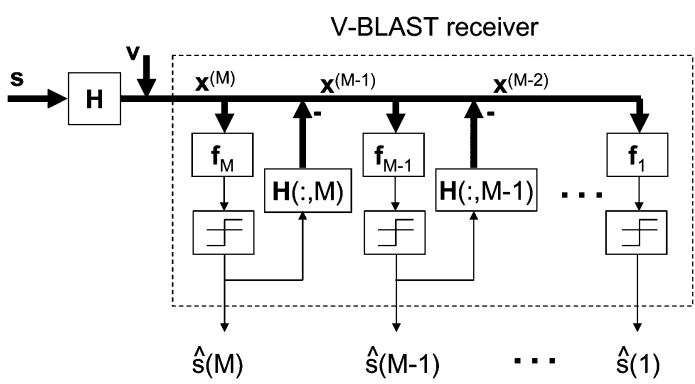

Fig. 1. V-BLAST scheme.

Recently, Jiang et al. [4] and independently Xu et al. [6] proposed an optimal linear precoder for the V-BLAST transmitter, by exploiting channel information. Fig. 2 shows this scheme. The linear precoder contains two parts.

1) MSE-equalizing precoder. The first part is a unitary precoder $\mathbf{P}$ which makes the mean square error (MSE) of each symbol stream in Fig. 2

$$
E|\mathbf{s}(k)-\hat{\mathbf{s}}(k)|^{2}
$$

identical for all $k$. Since it equalizes the MSE of all the symbol streams, we call this precoder the "MSE-equalizing precoder." The equalized MSE becomes the geometric mean of the original MSEs of the V-BLAST system without precoders. No bit loading is required because all of the symbol streams have the same MSE. Thus, the MSE-equalizing precoder can be viewed as an alternative to the bit-loading method. This will be further elaborated in Section IV-B.

2) Powerloading precoder. The second part of the precoder performs power loading. It is the same as the singular value decomposition (SVD ) diversity techniques [15], which decompose the channel matrix $\mathbf{H}$ by singular value decomposition (SVD) and obtain

$$
\mathbf{H}=\mathbf{U} \boldsymbol{\Lambda} \mathbf{V}^{\dagger} \text {. }
$$

The unitary matrix $\mathbf{V}$ is used as a linear precoder and different power is allocated on each eigenmode of the channel matrix by the diagonal matrix $\Gamma$ in Fig. 2. The details will be reviewed in Section IV-D.

In [4] and [6], only frequency-flat channels are considered. In this paper, we consider frequency-selective channels for the most part. Such channels are characterized by a transfer matrix $\mathbf{H}(z)$ which has memory. There are many different ways to equalize a frequency-selective MIMO channel. In [12] and [13], a finite-length minimum mean-square error-decision feedback 


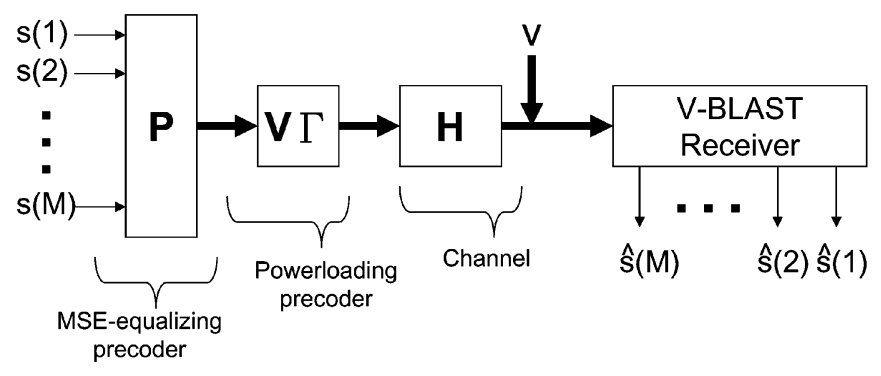

Fig. 2. Precoded V-BLAST scheme.

equalizer (MMSE-DFE) is introduced to equalize frequency-selective MIMO channels. It contains a feedforward FIR filter and a feedback FIR filter. In [14], the frequency-selective MIMO channel is converted into a parallel collection of frequency-flat MIMO channels by OFDM. The OFDM transmitter implements inverse discrete Fourier transform (IDFT) on every $K$ symbols of each antenna independently. To mitigate the ISI, each block of $K$ symbols is preceded by a cyclic prefix. This cyclic prefix converts the linear convolutions of the channels into circular convolutions. Therefore, the discrete Fourier transform (DFT) is performed at the receiver to convert the circulant convolutions into constant multipliers. Thus, the frequency-selective MIMO channels are converted into block MIMO channels. The equalizers for the block MIMO channels, such as V-BLAST [10] or linear MMSE equalizer, can be further applied to equalize these block MIMO channels.

Outline. The main contribution of this paper is that we extend the precoded V-BLAST system in [4] and [6] to frequency-selective MIMO channels. Two types of extensions are considered. One of them uses the FIR-based transceiver similar to [12] and [13]. The other uses redundancy at the transmitter, such as zero padding and cyclic prefixing. Numerical experiments show that the proposed precoded V-BLAST systems have better BER performances compared to many existing schemes which use channel information at the transmitter and receiver as described in Section VII. Since the existing transceivers with channel information available at the transmitters perform bit loading, the improvement comes from the fact that the MSE-equalizing precoder has better performance than the bit-loading method as we point out in Section IV-B.

The rest of the paper is organized as follows. In Section II, the GMD transceiver, which is the zero-forcing version of the precoded V-BLAST system, will be reviewed. In Sections III and IV, we review the V-BLAST system and the corresponding optimal precoder. In Section V, we extend the MSE-equalizing precoder and the V-BLAST system to the frequency-selective MIMO channel using FIR-based equalization. In Section VI, we extend the system for the case of transmitters with zero padding and cyclic prefix. In Section VII, we compare many different precoded communication systems based on numerical simulation. The detailed conclusions are presented in that section. Finally, Section VIII concludes the paper.

Notations. Matrices are denoted by capital letters in boldface. Vectors are denoted by lowercase letters in boldface. For convenience, MATLAB index notations for matrices will be used throughout this paper. For example, $\mathbf{H}(:, k:$ end) denotes the $k$ th though the last column of $\mathbf{H} ; \mathbf{H}(k,:)$ denotes the $k$ the row of the matrix $\mathbf{H}$; and $(\mathbf{Q v})(k)$ denotes the $k$ th element of vector Qv. Superscript ${ }^{\dagger}$ denotes the transpose conjugation. The determinant of a square matrix $\mathbf{A}$ is denoted by $\operatorname{det}(\mathbf{A})$. The notation $\otimes$ denotes the Kronecker product [21]. The notation $(x)^{+}$is defined as

$$
(x)^{+}= \begin{cases}x, & \text { if } x>0 \\ 0, & \text { otherwise } .\end{cases}
$$

A $N \times M$ matrix $\mathbf{P}$ satisfying $\mathbf{P}^{\dagger} \mathbf{P}=\mathbf{I}_{M}$ will be called unitary. Note that this requires $N \geq M$ and $\mathbf{P P}^{\dagger} \neq \mathbf{I}_{N}$ unless $N=M$.

\section{REVIEW OF THE GMD TRANSCEIVER}

Before we review the MMSE V-BLAST and its optimal precoder, we first review its zero-forcing version, namely the geometric mean decomposition (GMD) transceiver introduced in [3] and [8]. Fig. 3 shows this transceiver schematically, where s is the $M \times 1$ transmitted signal, $\mathbf{H}$ is an $N \times M$ channel matrix, and $\mathbf{v}$ is the channel noise. We assume $\mathbf{s}$ and $\mathbf{v}$ are zero mean and

$$
E\left(\begin{array}{c}
\mathbf{s} \\
\mathbf{v}
\end{array}\right)\left(\begin{array}{c}
\mathbf{s} \\
\mathbf{v}
\end{array}\right)^{\dagger}=\left(\begin{array}{cc}
\frac{1}{\alpha} \mathbf{I}_{M} & \mathbf{0} \\
\mathbf{0} & \mathbf{I}_{N}
\end{array}\right)
$$

where $\alpha$ is the noise-to-signal ratio. Furthermore, we assume $N \geq M$ in order to recover $M$ transmitted symbols from $N$ received signals. The matrices $\mathbf{P}$ and $\mathbf{Q}$ are unitary matrices which convert the channel $\mathbf{H}$ into a triangular matrix $\mathbf{R}$ with identical diagonal elements. They can be obtained by a process called the geometric mean decomposition (GMD) introduced by Jiang et al. [11]. According to this, for $N \geq M$, the $N \times M$ matrix $\mathbf{H}$ can be written in the form

$$
\mathbf{H}=\mathbf{Q R P}^{\dagger}
$$

where $\mathbf{Q}$ is a $N \times M$ unitary matrix and $\mathbf{P}$ is a $M \times M$ unitary matrix so that $\mathbf{R}$ is $M \times M$ triangular with identical diagonal elements. More specifically

$$
\mathbf{R}(k, k)=\operatorname{det}\left(\mathbf{H}^{\dagger} \mathbf{H}\right)^{(1 / 2 M)} \text { for } 1 \leq k \leq M .
$$

Thus, the channel has been converted into a triangular matrix. The input-output relationship then becomes

$$
\mathbf{x}=\mathbf{R} \mathbf{s}+\mathbf{Q}^{\dagger} \mathbf{v} .
$$

Thanks to the triangular structure of $\mathbf{R}$, a simple decision feedback algorithm can be applied to decode $\mathbf{s}$. The DFE block in Fig. 3 performs the following zero-forcing algorithm to decode $\mathbf{s}$ from $\mathrm{x}$

$$
\begin{aligned}
& \begin{array}{l}
\mathbf{x}^{(M)}=\mathbf{x} \\
\text { for } k=M:-1: 1 ; \\
\qquad \hat{\mathbf{s}}(k) \leftarrow C\left[(\mathbf{R}(k, k))^{-1} \mathbf{x}^{(k)}(k)\right] ; \\
\mathbf{x}^{(k-1)}(1: k-1) \leftarrow \mathbf{x}^{(k)}(1: k-1) \\
\quad-\mathbf{R}(1: k-1, k) \hat{\mathbf{s}}(k) ;
\end{array}
\end{aligned}
$$

end

where $C[\cdot]$ denotes the slicer. As in many analyses of decision feedback systems, we assume there is no error propagation. That 


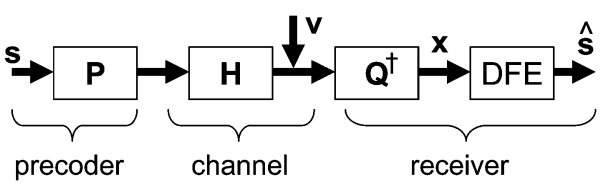

Fig. 3. GMD transceiver scheme.

is, when decoding the $k$ th symbol $\mathbf{s}(k)$, the previous decisions $\hat{\mathbf{s}}(i), i=M, M-1, \ldots, k+1$ are assumed to be correct. According to the above algorithm and using the above assumption $\hat{\mathbf{s}}(i)=\mathbf{s}(i)$ for $i=k+1, \ldots, M, \mathbf{x}^{(k)}(1: k)$ can be expressed as

$$
\begin{aligned}
\mathbf{x}^{(k)}(1: k) & =\mathbf{x}(1: k)-\sum_{i=k+1}^{M} \mathbf{R}(1: k, i) \hat{\mathbf{s}}(i) \\
& =\mathbf{R}(1: k, 1: k) \mathbf{s}(1: k)+\left(\mathbf{Q}^{\dagger} \mathbf{v}\right)(1: k) .
\end{aligned}
$$

Substituting the above equation into the algorithm, one can obtain

$$
\hat{\mathbf{s}}(k)=C\left[\mathbf{s}(k)+(\mathbf{R}(k, k))^{-1}\left(\mathbf{Q}^{\dagger} \mathbf{v}\right)(k)\right] .
$$

When the noise $\mathbf{v}$ is zero, the estimation is exactly $\mathbf{s}(k)$. Therefore, this is a zero-forcing decoder. The mean square error (MSE) of this system can be expressed as

$$
\begin{aligned}
& E\left|(\mathbf{R}(k, k))^{-1} \mathbf{x}^{(k)}(k)-\mathbf{s}(k)\right|^{2} \\
= & E\left|(\mathbf{R}(k, k))^{-1}\left(\mathbf{Q}^{\dagger} \mathbf{v}\right)(k)\right|^{2}=(\mathbf{R}(k, k))^{-2} \\
= & \operatorname{det}\left(\mathbf{H}^{\dagger} \mathbf{H}\right)^{-(1 / M)}
\end{aligned}
$$

for $k=1,2, \ldots, M$, where the last equality comes from (2).

To summarize, the GMD transceiver uses unitary matrices computed from the geometric mean decomposition to convert the channel matrix $\mathbf{H}$ into a triangular matrix $\mathbf{R}$ with identical diagonal elements. Then, the triangular channel matrix $\mathbf{R}$ can be equalized by the DFE. Due to the identical diagonal elements of $\mathbf{R}$, all symbol streams have identical MSE $\operatorname{det}\left(\mathbf{H}^{\dagger} \mathbf{H}\right)^{(-1 / M)}$. The overall system can be viewed as $M$ parallel SISO AWGN channels with identical noise variance which is equal to $\operatorname{det}\left(\mathbf{H}^{\dagger} \mathbf{H}\right)^{(-1 / M)}$.

In [5], the GMD transceiver has been applied for asymmetric UWB links. It has the best BER performance among the transceivers tested in [5]. The drawback of the GMD transceiver is that the MSE $\operatorname{det}\left(\mathbf{H}^{\dagger} \mathbf{H}\right)^{(-1 / M)}$ becomes very large when one of the singular values of $\mathbf{H}$ is small, and goes to infinity when $\mathbf{H}$ has a null. Since every SISO subchannel shares the same MSE, this causes a serious degradation in performance. The amplification of the noise comes from the zero-forcing design of the DFE receiver. It can be avoided if the linear minimum mean square error (LMMSE) estimator is used. We shall return to this in Section IV.

\section{REVIEW OF THE V-BLAST SYSTEM}

In this section, we review the V-BLAST system proposed in [10]. The V-BLAST system is a decision feedback equalizer in the spatial domain. Fig. 1 shows the V-BLAST scheme, where $\mathbf{s}$ is the $M \times 1$ transmitted signal, $\mathbf{H}$ is an $N \times M$ channel matrix, $\mathbf{v}$ is the channel noise, and $\mathbf{x}^{(M)}=\mathbf{H} \mathbf{s}+\mathbf{v}$ is the $N \times 1$ received signal. We assume $\mathbf{s}$ and $\mathbf{v}$ have the same statistics as in (1) and $N \geq M$. The V-BLAST receiver decodes each symbol by the LMMSE estimator and then feeds back the decoded symbol to cancel its interference with other symbols. This process repeats until all of the symbols are decoded. The decoding order can be further optimized. For convenience, we assume the decoding order is $(M, M-1, \ldots, 1)$ as shown in Fig. 1. This sequence can be easily modified by inserting a permutation matrix in Fig. 1 between the channel $\mathbf{H}$ and the V-BLAST decoder.

In Fig. $1, \mathbf{f}_{k}$ is an $1 \times N$ vector such that $\mathbf{f}_{k} \mathbf{x}^{(k)}$ is the LMMSE estimator of the $k$ th element of $\mathbf{s}$ (denoted as $\mathbf{s}(k)$ ) based on the input $\mathbf{x}^{(k)}$. It can be found from the following optimization problem:

$$
\min _{\mathbf{f}_{k}} E\left|\mathbf{f}_{k} \mathbf{x}^{(k)}-\mathbf{s}(k)\right|^{2} .
$$

After this estimation, $\mathbf{f}_{k} \mathbf{x}^{(k)}$ is sent to the slicer and fedback to cancel the interference caused by $\mathbf{s}(k)$. That is

$$
\mathbf{x}^{(k-1)}=\mathbf{x}^{(k)}-\mathbf{H}(:, k) \hat{\mathbf{s}}(k)
$$

where $\hat{\mathbf{s}}(k)=C\left[\mathbf{f}_{k} \mathbf{x}^{(k)}\right]$ and $C[\cdot]$ denote the slicer. As in the previous analysis of decision feedback systems, we assume there is no error propagation. That is, when considering the $k$ th input $\mathbf{x}^{(k)}$, we assume $\hat{\mathbf{s}}(i)=\mathbf{s}(i), i=M, M-1, \ldots, k+1$. Therefore

$$
\begin{aligned}
\mathbf{x}^{(k)} & =\mathbf{x}^{(M)}-\sum_{i=k+1}^{M} \mathbf{H}(:, i) \mathbf{s}(i) \\
& =\mathbf{H} \mathbf{s}+\mathbf{v}-\mathbf{H}(:, k+1: M) \mathbf{s}(k+1: M) \\
& =\underbrace{\mathbf{H}(:, 1: k)}_{\text {Call this } \mathbf{A}_{k}} \mathbf{s}(1: k)+\mathbf{v} .
\end{aligned}
$$

From the above equation, one can derive $\mathbf{f}_{k}$ and the corresponding MSE by the orthogonality principle and obtain

$$
\begin{aligned}
\mathbf{f}_{k} & =\left(\left(\mathbf{A}_{k}^{\dagger} \mathbf{A}_{k}+\alpha \mathbf{I}_{k}\right)^{-1} \mathbf{A}_{k}^{\dagger}\right)(k,:) \\
E\left|\mathbf{s}(k)-\mathbf{f}_{k} \mathbf{x}^{(k)}\right|^{2} & =\left(\mathbf{A}_{k}^{\dagger} \mathbf{A}_{k}+\alpha \mathbf{I}_{k}\right)^{-1}(k, k)
\end{aligned}
$$

where $\mathbf{A}(k,:)$ denotes the $k$ th row of the matrix $\mathbf{A}$. However, this direct computation is very complex. A matrix inversion has to be computed for each $\mathbf{f}_{k}$. A fast way to compute these is by using the QR algorithm [1]. Thus, we first compute the following QR decomposition:

$$
\left(\begin{array}{c}
\mathbf{H} \\
\sqrt{\alpha} \mathbf{I}_{M}
\end{array}\right)=\mathbf{Q R}
$$

where $\mathbf{Q}$ is an $(N+M) \times M$ unitary matrix and $\mathbf{R}$ is $M \times M$ and upper triangular with positive diagonal elements. Observing the first $k$ columns of the equation above, we obtain the following QR decomposition:

$$
\underbrace{\left(\begin{array}{c}
\mathbf{A}_{k} \\
\sqrt{\alpha} \mathbf{I}_{k} \\
\mathbf{0}_{(M-k) \times k}
\end{array}\right)}_{\text {Call this } \mathbf{H}_{k} .}=\mathbf{Q}\left(\begin{array}{c}
\mathbf{R}(1: k, 1: k) \\
\mathbf{0}_{(M-k) \times k}
\end{array}\right) .
$$


The solution of $\mathbf{f}_{k}$ in (3) can be rewritten as $\mathbf{f}_{k}=$ $\left(\left(\mathbf{H}_{k}^{\dagger} \mathbf{H}_{k}\right)^{-1} \mathbf{H}_{k}^{\dagger}\right)(k, 1: \quad N)$. Substituting the $\mathrm{QR}$ expression of $\mathbf{H}_{k}$ above, we obtain

$$
\begin{aligned}
\mathbf{f}_{k} & =\left((\mathbf{R}(1: k, 1: k))^{-1}(\mathbf{Q}(1: N,:))^{\dagger}\right)(k,:) \\
& =(\mathbf{R}(k, k))^{-1}(\mathbf{Q}(1: N, k))^{\dagger} .
\end{aligned}
$$

The last equality comes from the fact that $\mathbf{R}(1: k, 1: k)$ is an upper triangular matrix. Similarly, the MSE in (4) can be rewritten as $\left(\mathbf{H}_{k}^{\dagger} \mathbf{H}_{k}\right)^{-1}(k, k)$. Substituting the $\mathrm{QR}$ expression of $\mathbf{H}_{k}$ into (4), we obtain

$$
E\left|\mathbf{s}(k)-\mathbf{f}_{k} \mathbf{x}^{(k)}\right|^{2}=(\mathbf{R}(k, k))^{-2} .
$$

Thus, by computing one QR decomposition in (5), all of the LMMSE estimator coefficients can be obtained by (6) and the MSE can be obtained by (7). This is more efficient than the direct computation in (3) and (4). The decoding order can be optimized by the following variation of the $\mathrm{QR}$ algorithm:

$$
\left(\begin{array}{c}
\mathbf{H} \\
\sqrt{\alpha} \mathbf{I}_{M}
\end{array}\right)=\mathrm{QRE}
$$

where $\mathbf{E}$ is a permutation chosen so that $\mathbf{R}(k, k)$ decreases. Thus, the symbol with smaller MSE can be decoded first. This reduces error propagation.

The output of the $k$ th LMMSE estimator is given by

$$
\begin{aligned}
\mathbf{f}_{k} \mathbf{x}^{(k)} & =\mathbf{f}_{k}(:, k) \mathbf{s}(k)+\underbrace{\mathbf{f}_{k}(\mathbf{H}(:, 1: k-1) \mathbf{s}(1: k-1)+\mathbf{v})}_{A W G N}, \\
k & =1,2, \ldots, M .
\end{aligned}
$$

The transmitted symbol $\mathbf{s}(k)$ is assumed to be an i.i.d. Gaussian variable. Therefore, the error, which is the sum of interferences from other symbols and the channel noise, is also AWGN. Thus, the V-BLAST system converts the MIMO channel into the above $M$ parallel single-input single-output (SISO) AWGN channels. Since the channel coding is done independently in each parallel SISO channel, we define the capacity of the V-BLAST system as the sum of the capacities of these SISO channels. The SNR of the $k$ th SISO channel can be expressed as

$$
\rho_{k}=\frac{E\left|\mathbf{f}_{k}(:, k) \mathbf{s}(k)\right|^{2}}{E\left|\mathbf{f}_{k}(\mathbf{H}(:, 1: k-1) \mathbf{s}(1: k-1)+\mathbf{v})\right|^{2}},
$$

for $1 \leq k \leq M$. It has been proven in [4] that

$$
1+\rho_{k}=\frac{1}{\alpha}(\mathbf{R}(k, k))^{2} .
$$

Therefore, the capacity of the V-BLAST system can be expressed as

$$
\begin{aligned}
C_{v \text { blast }} & =\sum_{k=1}^{M} \log \left(1+\rho_{k}\right)=\log \prod_{k=1}^{M} \frac{1}{\alpha}(\mathbf{R}(k, k))^{2} \\
& =\log \left(\operatorname{det}\left(\frac{1}{\alpha} \mathbf{H}^{\dagger} \mathbf{H}+\mathbf{I}_{M}\right)\right) \\
& =\log \left(\operatorname{det}\left(\frac{1}{\alpha} \mathbf{H H}^{\dagger}+\mathbf{I}_{N}\right)\right) .
\end{aligned}
$$

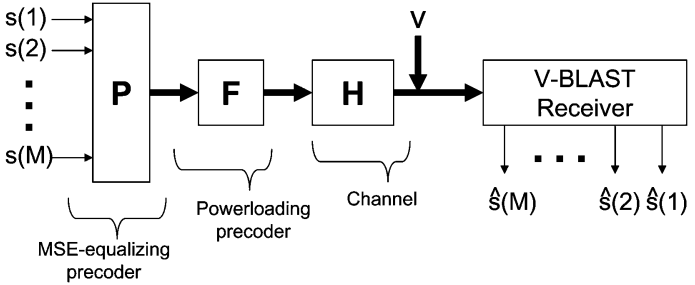

Fig. 4. Precoded V-BLAST scheme.

The third equality can be verified by taking determinant of the equality $\mathbf{H}^{\dagger} \mathbf{H}+\alpha \mathbf{I}_{M}=\mathbf{R}^{\dagger} \mathbf{R}$. The capacity of the MIMO channel can be expressed as [18]

$$
C_{\text {mimo }}=\max _{(1 / M) \operatorname{tr}\left(\mathbf{R}_{s}\right)=(1 / \alpha)} \log \operatorname{det}\left(\mathbf{H R}_{s} \mathbf{H}^{\dagger}+\mathbf{I}_{N}\right)
$$

where $\mathbf{R}_{s}=E\left[\mathbf{s s}^{\dagger}\right]$ and the constraint ensures that the average transmitted power is $1 / \alpha$. Therefore, the capacity of the V-BLAST system can be viewed as the capacity of the MIMO channel with the restriction $\mathbf{R}_{s}=(1 / \alpha) \mathbf{I}_{M}$. This means there is no capacity loss after converting the MIMO channel into $M$ parallel SISO channels. This fact has been observed in [4]. To achieve the channel capacity with the V-BLAST system, one can further apply a powerloading precoder so that $\mathbf{R}_{s}$ is optimized. We will discuss the powerloading precoder in Section IV

\section{REVIEW OF THE UCD SYSTEM}

In this section, we review the uniform channel decomposition (UCD) system introduced in [4] and [6]. It can be viewed as a V-BLAST system with an optimal precoder derived from channel information. Fig. 4 shows this system schematically. The optimal precoder contains two parts. The first part is a unitary linear precoder $\mathbf{P}$ whose purpose is to force each symbol stream to have identical MSE at the receiver. It can be viewed as an alternative to bit loading. We call this precoder "MSE-equalizing precoder" throughout this paper. The second part is the matrix $\mathbf{F}$ in Fig. 4. It allocates transmitted power to each eigenmode of the channel matrix $\mathbf{H}$ to minimize the MSE at the receiver while fixing the transmitting power. We call this precoder "powerloading precoder" throughout the paper.

\section{A. MSE-Equalizing Precoder for V-BLAST System}

The idea of the MSE-equalizing precoder has been introduced in [4] and [6]. Consider the MSE equalizer for the channel $\mathbf{H}$ only, ignoring the powerloading precoder $\mathbf{F}$ in Fig. 4. From (7), the MSE is related to the diagonal elements of the matrix $\mathbf{R}$ obtained from the QR decomposition in (5). Therefore, the MSE-equalizing precoder $\mathbf{P}$ is designed so that the precoded channel HP has a corresponding upper triangular matrix with identical diagonal elements. This can be obtained by the following geometric mean decomposition (GMD) reviewed in Section II:

$$
\left(\begin{array}{c}
\mathbf{H} \\
\sqrt{\alpha} \mathbf{I}_{M}
\end{array}\right)=\mathbf{Q}_{1} \mathbf{R}_{1} \mathbf{P}^{\dagger}
$$


where $\mathbf{Q}_{1}$ and $\mathbf{P}$ are unitary matrices and $\mathbf{R}_{1}$ is $M \times M$ and upper triangular. Furthermore, $\mathbf{R}_{1}(k, k)$ is identical for all $k$ and it can be expressed as

$$
\mathbf{R}_{1}(k, k)=\operatorname{det}\left(\mathbf{H}^{\dagger} \mathbf{H}+\alpha \mathbf{I}_{M}\right)^{(1 / 2 M)}=\left(\prod_{i=1}^{M} \mathbf{R}(i, i)\right)^{(1 / M)}
$$

for all $k$, where $\mathbf{R}$ is from the original $\mathrm{QR}$ decomposition in (5). Equation (11) can be easily verified by taking determinant of both sides in

$$
\mathbf{H}^{\dagger} \mathbf{H}+\alpha \mathbf{I}_{M}=\mathbf{P} \mathbf{R}_{1}^{\dagger} \mathbf{R}_{1} \mathbf{P}^{\dagger}=\mathbf{R}^{\dagger} \mathbf{R} .
$$

By this decomposition, $\mathbf{R}_{1}(k, k)$ is equal to the geometric mean of $\{\mathbf{R}(i, i)\}_{i=1}^{M}$ for $k=1,2, \ldots, M$. Now, let $\mathbf{P}$ be the precoder. Substituting the precoded channel $\mathbf{H P}$ and using the GMD in (10), the QR decomposition corresponding to (5) can be obtained by

$$
\begin{aligned}
\left(\begin{array}{c}
\mathbf{H P} \\
\sqrt{\alpha} \mathbf{I}_{M}
\end{array}\right) & =\left(\begin{array}{cc}
\mathbf{I}_{N} & \mathbf{0}_{N \times M} \\
\mathbf{0}_{M \times N} & \mathbf{P}^{\dagger}
\end{array}\right)\left(\begin{array}{c}
\mathbf{H} \\
\sqrt{\alpha} \mathbf{I}_{M}
\end{array}\right) \mathbf{P} \\
& =\underbrace{\left(\begin{array}{cc}
\mathbf{I}_{N} & \mathbf{0}_{N \times M} \\
\mathbf{0}_{M \times N} & \mathbf{P}^{\dagger}
\end{array}\right) \mathbf{Q}_{1}}_{\text {unitary }} \mathbf{R}_{1}^{\underbrace{\mathbf{P}^{\dagger} \mathbf{P}}_{\mathbf{I}_{M}}} .
\end{aligned}
$$

Thus, by (7), with the above precoder, the MSE corresponding to the $k$ th element is

$$
\begin{aligned}
E\left|\mathbf{f}_{k} \mathbf{x}^{(k)}-\mathbf{s}(k)\right|^{2} & =\left(\mathbf{R}_{1}(k, k)\right)^{-2} \\
& =\operatorname{det}\left(\mathbf{H}^{\dagger} \mathbf{H}+\alpha \mathbf{I}_{M}\right)^{-(1 / M)}
\end{aligned}
$$

for all $k$. It is equal to the geometric mean of the MSEs of the original V-BLAST system.

\section{B. Comparison of the V-BLAST System With MSE-Equalizing Precoder and V-BLAST System With Optimal Bit Loading}

Bit loading is a technique that uses different sizes of constellation among parallel subchannels so that the BERs among all subchannels are approximately equal. The MSE-equalizing precoder also results in approximately equal BERs among all subchannels by making the corresponding MSEs identical. Thus, the MSE-equalizing precoder can be viewed as an alternative to the bit-loading algorithm. To compare these two methods, we derive the closed-form expression for the bit transmission rate $(b$ bits per channel use) as a function of BER $P_{e}$ and noise-to-signal ratio $\alpha$ for both methods.

For a quadrature amplitude modulation (QAM) transmission over an additive white Gaussian (AWGN) channel with variance $\sigma_{k}^{2}$, in order to achieve BER $P_{e}$, the minimum distance in the constellation $D_{k}$ needs to satisfy [23, p. 371]

$2 Q\left(\frac{D_{k}}{2 \sigma_{k}}\right) \cong P_{e}$, where $Q(x)=\int_{x}^{\infty} \frac{1}{\sqrt{2 \pi}} \exp \left(\frac{-t^{2}}{2}\right) d t$.
Thus, the average power $P_{k}$ to transmit $b_{k}$ bits under BER $P_{e}$ can be expressed as

$$
\begin{aligned}
P_{k}= & \frac{1}{2^{b_{k} / 2}} D_{k}^{2} \\
& \times\left(\left(\frac{1}{2}\right)^{2}+\left(\frac{3}{2}\right)^{2}+\cdots+\left(\frac{2^{b_{k} / 2}-1}{2}\right)^{2}\right) \times 4 \\
= & \frac{2}{3} \sigma_{k}^{2} Q^{-1}\left(\frac{P_{e}}{2}\right)^{2}\left(2^{b_{k}-1}-1\right)
\end{aligned}
$$

where $Q^{-1}(\cdot)$ is the inverse function of $Q(\cdot)$. Applying the high bit rate assumption $2^{b_{k}} \gg 1$, we obtain

$$
b_{k} \cong \log \frac{P_{k}}{\sigma_{k}^{2}}-\log \frac{2}{3} Q^{-1}\left(\frac{P_{e}}{2}\right)^{2} .
$$

For the V-BLAST system with the MSE-equalizing precoder, in (12), the MSE is $\operatorname{det}\left(\mathbf{H}^{\dagger} \mathbf{H}+\alpha \mathbf{I}_{M}\right)^{-(1 / M)}$ and the transmitted power is $(1 / \alpha)$ for each symbol stream index $k$. We approximate the error by an AWGN source with a variance equal to the MSE of the error. Substituting $\sigma_{k}^{2} \cong \operatorname{det}\left(\mathbf{H}^{\dagger} \mathbf{H}+\alpha \mathbf{I}_{M}\right)^{-1 / M}$ and $P_{k}=(1 / \alpha)$ into (14) and summing over $k$, we have the bit rate

$$
\begin{aligned}
b_{\mathrm{eq}} \cong & M\left(\log \frac{\frac{1}{\alpha}}{\operatorname{det}\left(\mathbf{H}^{\dagger} \mathbf{H}+\alpha \mathbf{I}_{M}\right)^{-(1 / M)}}\right. \\
& \left.\quad-\log \frac{2}{3} Q^{-1}\left(\frac{P_{e}}{2}\right)^{2}\right) \\
= & \log \operatorname{det}\left(\left(\frac{1}{\alpha}\right) \mathbf{H}^{\dagger} \mathbf{H}+\mathbf{I}_{M}\right)-M \log \frac{2}{3} Q^{-1}\left(\frac{P_{e}}{2}\right)^{2} .
\end{aligned}
$$

This approximation is only valid for high SNR.

In the case of the V-BLAST system with optimal bit loading, the optimal bit loading chooses $b_{k}, k=1,2, \ldots, M$ so that the total number of bits transmitted is maximized under fixed total power $(1 / M) \sum_{k=1}^{M} P_{k}=(1 / \alpha)$ and fixed BER $P_{e}$. Using the $P_{k}$ in (13) and the high bit rate assumption $2^{b_{k}} \gg 1$, the optimal bit-loading problem can be formulated as

$$
\begin{aligned}
& \max _{b_{k}} \sum_{k=1}^{M} b_{k}=b_{b l} \\
& \text { subject to }\left(\frac{1}{M}\right) \sum_{k=1}^{M} \frac{2}{3} \sigma_{k}^{2} Q^{-1}\left(\frac{P_{e}}{2}\right)^{2} 2^{b_{k}} \leq\left(\frac{1}{\alpha}\right)
\end{aligned}
$$

where $b_{b l}$ is the total number of bits to be transmitted per channel use. By the arithmetic mean geometric mean (AM-GM) inequality, we have

$$
\begin{aligned}
\left(\frac{1}{\alpha}\right) & \geq\left(\frac{1}{M}\right) \sum_{k=1}^{M} \frac{2}{3} \sigma_{k}^{2} Q^{-1}\left(\frac{P_{e}}{2}\right)^{2} 2^{b_{k}} \\
& \geq \frac{2}{3}\left(\prod_{k=1}^{M} \sigma_{k}^{2}\right)^{(1 / M)} Q^{-1}\left(\frac{P_{e}}{2}\right)^{2} 2^{\left(b_{b l} / M\right)} .
\end{aligned}
$$


Thus, the upperbound on the bit rate $b_{b l}$ can be expressed as

$$
b_{b l} \leq M \log \frac{\frac{1}{\alpha}}{\prod_{k=1}^{M} \sigma_{k}^{2}}-\log \frac{2}{3} Q^{-1}\left(\frac{P_{e}}{2}\right)^{2} .
$$

Again, we approximate the error by an AWGN source with a variance equal to the MSE of the error. Substituting the MSE of the V-BLAST system $\sigma_{k}^{2} \cong(\mathbf{R}(k, k))^{-2}$ obtained from (7) into the above equation and using the equality in (11), one can obtain

$$
\begin{aligned}
b_{b l} \leq & M\left(\log \frac{\frac{1}{\alpha}}{\left(\prod_{k=1}^{M}(\mathbf{R}(k, k))^{-2}\right)^{(1 / M)}}\right. \\
& \left.\quad-\log \frac{2}{3} Q^{-1}\left(\frac{P_{e}}{2}\right)^{2}\right) \\
& =\log \operatorname{det}\left(\left(\frac{1}{\alpha}\right) \mathbf{H}^{\dagger} \mathbf{H}+\mathbf{I}_{M}\right)-M \log \frac{2}{3} Q^{-1}\left(\frac{P_{e}}{2}\right)^{2} \\
= & b_{\text {eq }} .
\end{aligned}
$$

The upperbound is equal to the bit rate of the MSE-equalizing method $b_{\text {eq }}$. It can be achieved if and only if $b_{k}$ satisfies

$$
b_{k}=\frac{b_{b l}}{M}+\log _{2}\left(\prod_{j=1}^{M} \sigma_{j}^{2}\right)^{(1 / M)}-\log _{2} \sigma_{k}^{2}
$$

for all $k=1,2, \ldots, M$ so that the equality holds in (15). This requires that the right-hand side of the above equation is a positive integer for all $k$. In practice, optimality is therefore lost. Therefore, the BER performance of the system with the MSEequalizing precoder is better than that of the system with optimal bit loading. The discussion above is confined to using different sizes of QAM constellations. However, for bit loading, using coding and a combination of different constellations, it is not clear whether the MSE-equalizing precoder has better BER performance than bit loading.

\section{Comparison of V-BLAST System With MSE-Equalizing \\ Precoder and SVD-Based System With Optimal Bit Loading}

In this subsection, the SVD-based system with optimal bit loading [16] is compared to the V-BLAST system with MSEequalizing precoder by similar analysis as in Section IV-B. The channel matrix $\mathbf{H}$ can be decomposed by the following SVD $\mathbf{H}=\mathbf{U} \mathbf{\Lambda} \mathbf{V}^{\dagger}$, where $\mathbf{U}$ and $\mathbf{V}$ are unitary and $\boldsymbol{\Lambda}$ is positive real diagonal. By letting $\mathbf{V}$ be the precoder and $\mathbf{U}^{\dagger}$ be the postfilter, the channel can be converted into parallel SISO channels with channel gain $\boldsymbol{\Lambda}(k, k)$, and unit noise variance for $k=$ $1,2, \ldots, M$. We can apply an LMMSE estimator

$$
\frac{\Lambda(k, k)}{(\Lambda(k, k))^{2}+\alpha}
$$

to the $k$ th SISO channel and the corresponding MSE becomes $\left((\boldsymbol{\Lambda}(k, k))^{2}+\alpha\right)^{-1}$. Again, approximating the error by an
AWGN source and substituting the above MSE for $\sigma_{k}^{2}$ in (16), we obtain

$$
\begin{aligned}
& b_{\mathrm{svd}} \leq M\left(\log \frac{\frac{1}{\alpha}}{\prod_{k=1}^{M}\left((\Lambda(k, k))^{2}+\alpha\right)^{-(1 / M)}}\right. \\
&\left.\quad-\log \frac{2}{3} Q^{-1}\left(\frac{P_{e}}{2}\right)^{2}\right) \\
&=\log \operatorname{det}\left(\left(\frac{1}{\alpha}\right) \mathbf{H}^{\dagger} \mathbf{H}+\mathbf{I}_{M}\right)-M \log \frac{2}{3} Q^{-1}\left(\frac{P_{e}}{2}\right) \\
&=b_{\text {eq }} .
\end{aligned}
$$

This bound is achievable if and only if (17) can be satisfied. This requires that the right-hand side of (17) be a positive integer for all $k$. In practice, optimality is therefore lost. The above upperbound is, however, identical to the bit rate of the V-BLAST system with the MSE-equalizing precoder derived in Section IV-B. Therefore, the V-BLAST system with the MSE-equalizing precoder has better BER performance than the SVD-based optimal bit loading system. Again, the above discussion is confined to bit loading using different sized QAM constellations. For bit loading using coding and a combination of different constellations, it is not clear whether the V-BLAST system with the MSE-equalizing precoder still has better performance than the SVD scheme with bit loading. Also, this analysis is based on the assumption that there is no error propagation. Error propagation degrades the performance of the V-BLAST-based system while the SVD scheme has no such problems.

\section{Power Loading Precoder for V-BLAST System}

The powerloading precoder has been proposed with the MSEequalizing precoder in both [4] and [6]. After the MSE-equalizing precoder is applied, the MSE in (12) can be further minimized by adding another precoder $\mathbf{F}$ as indicated in Fig. 4. The optimization problem can be written as

$$
\min _{\operatorname{tr}\left(\mathbf{F} \mathbf{F}^{\dagger}\right) \leq M} \operatorname{det}\left(\mathbf{F}^{\dagger} \mathbf{H}^{\dagger} \mathbf{H F}+\alpha \mathbf{I}_{M}\right)^{-(1 / M)}
$$

where the constraint ensures that the average transmitted power is $(1 / \alpha)$. To find this optimal precoder, we first compute the following SVD of the channel $\mathbf{H}=\mathbf{U} \mathbf{\Lambda} \mathbf{V}^{\dagger}$, where $\mathbf{U}$ and $\mathbf{V}$ are unitary and $\boldsymbol{\Lambda}$ is $M \times M$ and diagonal. Without loss of generality, this precoder can be expressed as $\mathbf{F}=\mathbf{V} \boldsymbol{\Gamma}$, where $\Gamma$ is an arbitrary $M \times M$ matrix. Thus, the power constraint can be rewritten as $\operatorname{tr}\left(\mathbf{F} \mathbf{F}^{\dagger}\right)=\operatorname{tr}\left(\boldsymbol{\Gamma} \boldsymbol{\Gamma}^{\dagger}\right) \leq M$. Substituting the precoded channel $\mathbf{H V} \Gamma^{\dagger}$ into the MSE in (12) and applying the SVD, we obtain

$$
\begin{aligned}
& E\left|\mathbf{f}_{k} \mathbf{x}^{(k)}-\mathbf{s}(k)\right|^{2} \\
& =\operatorname{det}\left(\boldsymbol{\Gamma}^{\dagger} \boldsymbol{\Lambda}^{\dagger} \boldsymbol{\Lambda} \boldsymbol{\Gamma}+\alpha \mathbf{I}_{M}\right)^{-(1 / M)} \\
& =\operatorname{det}\left(\boldsymbol{\Lambda} \boldsymbol{\Gamma} \boldsymbol{\Gamma}^{\dagger} \boldsymbol{\Lambda}^{\dagger}+\alpha \mathbf{I}_{N}\right)^{-(1 / M)} \\
& \quad \geq\left(\prod_{i=1}^{M}\left(\boldsymbol{\Gamma} \boldsymbol{\Gamma}^{\dagger}\right)(i, i)(\boldsymbol{\Lambda}(i, i))^{2}+\alpha\right)^{-(1 / M)} .
\end{aligned}
$$


The Hadamard's inequality is used to obtain the last inequality [22]. Define $\boldsymbol{\Gamma} \Gamma^{\dagger}=\boldsymbol{\Phi}$. The equality holds if and only if $\boldsymbol{\Phi}$ is a diagonal matrix. For any $\boldsymbol{\Phi}$ which satisfies the power constraint $\operatorname{tr}(\boldsymbol{\Phi})=\operatorname{tr}\left(\boldsymbol{\Gamma} \boldsymbol{\Gamma}^{\dagger}\right) \leq M$, the diagonal matrix $\operatorname{diag}\left(\{\boldsymbol{\Phi}(i, i)\}_{i=1}^{M}\right)$ also satisfies the power constraint because it has the same trace. Moreover, this diagonal matrix has smaller MSE as shown in (19). Therefore, to minimize the MSE, $\boldsymbol{\Phi}$ must be a diagonal matrix. The diagonal elements of the diagonal matrix $\Phi$ can be found by solving the following problem:

$$
\begin{aligned}
& \max _{\boldsymbol{\Phi}(i, i)>0} \prod_{i=1}^{M}\left(\boldsymbol{\Phi}(i, i)(\boldsymbol{\Lambda}(i, i))^{2}+\alpha\right) \\
& \text { subject to } \sum_{i=1}^{M} \boldsymbol{\Phi}(i, i)=M .
\end{aligned}
$$

One can solve this by the Lagrange multiplier method and obtain

$$
\Phi(i, i)=\left(a-\frac{\alpha}{(\Lambda(i, i))^{2}}\right)^{+}
$$

where $a$ is a positive real number so that $(1 / M) \sum_{i=1}^{M} \boldsymbol{\Phi}(i, i)=$ 1 . The solution is precisely the water filling method. This matrix diagonal $\boldsymbol{\Phi}$ can be simply implemented by choosing a diagonal $\Gamma$ so that

$$
\Gamma(i, i)=\sqrt{\boldsymbol{\Phi}(i, i)} .
$$

The overall powerloading precoder is $\mathbf{F}=\mathbf{V} \boldsymbol{\Gamma}$. It is the precoder which minimizes the MSE after the MSE-equalizing precoder in Section A is used. It allocates different transmitted power on each eigenmode of the channel matrix according to the SNR. The eigenmode with higher SNR gets larger transmitted power. The SVD-based system in Section IV-C can also apply powerloading [16]. The comparison of these two systems with powerloading is similar to the comparison performed in Section IV-C. The V-BLAST system with both MSE-equalizing and powerloading precoder still has a better bit-rate performance than the SVD-based system with both bit loading and power loading. The performances of the power-loading precoder are demonstrated in Section VII.

\section{E. Capacity Losslessness of the UCD System}

The capacity losslessness of the UCD system has been first proved by Jiang et al. in [4]. In Section III, we have discussed the capacity of the V-BLAST system. The UCD system is a V-BLAST system with the MSE-equalizing precoder $\mathbf{P}$ discussed in Section IV-A and the powerloading precoder VT discussed in Section IV-D. By substituting the precoded channel HVTP into the capacity of the V-BLAST system in (8), we have

$$
\begin{aligned}
C_{\mathrm{ucd}} & =\log \operatorname{det}\left(\mathbf{P}^{\dagger} \boldsymbol{\Gamma}^{\dagger} \mathbf{V}^{\dagger} \mathbf{H}^{\dagger} \mathbf{H V} \boldsymbol{\Gamma P} \frac{1}{\alpha}+\mathbf{I}_{M}\right) \\
& =\log \operatorname{det}\left(\boldsymbol{\Gamma}^{\dagger} \boldsymbol{\Lambda}^{\dagger} \boldsymbol{\Lambda} \boldsymbol{\Gamma} \frac{1}{\alpha}+\mathbf{I}_{M}\right)
\end{aligned}
$$

Since VT is the solution to the optimization problem in (18), the capacity can also be expressed as

$$
\begin{aligned}
C_{\mathrm{ucd}} & =\max _{(1 / M) \operatorname{tr}\left(\mathbf{F} \mathbf{F}^{\dagger}\right)=1} \log \operatorname{det}\left(\mathbf{F}^{\dagger} \mathbf{H}^{\dagger} \mathbf{H F}\left(\frac{1}{\alpha}\right)+\mathbf{I}_{M}\right) \\
& \left.=\max _{(1 / M) \operatorname{tr}(\mathbf{F F}}^{\dagger}\right)=1 \\
& =\max _{(1 / M) \operatorname{tr}\left(\mathbf{R}_{s}\right)=(1 / \alpha)} \log \operatorname{det}\left(\mathbf{H F F} \mathbf{F}^{\dagger} \mathbf{H}^{\dagger} \frac{1}{\alpha}+\mathbf{I}_{N}\right) \\
& =C_{\operatorname{mimo}},
\end{aligned}
$$

where $C_{\text {mimo }}$ is defined in (9). This shows that the capacity is preserved after converting the MIMO channel into $M$ parallel SISO channels by the UCD system as shown first in [4]. Moreover, these $M$ parallel SISO channels have identical MSE. This means that we can use the same constellation and the same coding technique on every SISO channel separately.

\section{PRECOdEd FIR V-BLAST FOR FREQUENCY-SELECTIVE MIMO CHANNELS}

In this section, the V-BLAST system and the UCD system are generalized to the case of the frequency-selective MIMO channel $\mathbf{H}(z)$. The FIR MMSE DFE for frequency-selective MIMO channels and the corresponding MSE-equalizing precoder are derived.

\section{A. FIR DFE for Frequency-Selective MIMO Channels}

The FIR MMSE DFE for frequency-selective MIMO channels has been introduced first by Al-Dhahir and Sayed [12]. We briefly derive it in a simpler way. The input-output relation of the frequency-selective MIMO channel can be expressed as

$$
\mathbf{x}_{i}=\sum_{k=0}^{L} \mathbf{H}_{k} \mathbf{s}_{i-k}+\mathbf{v}_{i}
$$

where $\mathbf{s}_{i}$ is the $M \times 1$ transmitted signal, $\mathbf{H}(z)=\mathbf{H}_{0}+\mathbf{H}_{1} z^{-1}+$ $\cdots+\mathbf{H}_{L} z^{-L}$ is the $L$ th order $N \times M$ frequency-selective MIMO channel, $\mathbf{v}_{i}$ is the $N \times 1$ channel noise, and $\mathbf{x}_{i}$ is the $N \times 1$ received signal. Furthermore, we assume the following statistics:

$$
E\left(\begin{array}{c}
\mathbf{s}_{i} \\
\mathbf{v}_{i}
\end{array}\right)\left(\begin{array}{c}
\mathbf{s}_{j} \\
\mathbf{v}_{j}
\end{array}\right)^{\dagger}=\delta(i-j)\left(\begin{array}{cc}
\frac{1}{\alpha} \mathbf{I}_{M} & \mathbf{0}_{M \times N} \\
\mathbf{0}_{N \times M} & \mathbf{I}_{N}
\end{array}\right)
$$

and $\mathbf{s}_{i}$ and $\mathbf{v}_{i}$ are zero-mean. Let $d$ be the order of the FIR DFE and $\Delta$ be the decision delay. At time $i$, the FIR DFE decodes $\mathbf{s}_{i-\Delta}$ based on the observed received signals $\mathbf{x}_{i}, \mathbf{x}_{i-1}, \ldots, \mathbf{x}_{i-d}$ along with the previous decoded signals $\mathbf{s}_{i-\Delta-1}, \mathbf{s}_{i-\Delta-2}, \ldots$. Again, we assume that the previous signals are correctly decoded. The observed received signals can be expressed as

$$
\left(\begin{array}{c}
\mathbf{x}_{i} \\
\mathbf{x}_{i-1} \\
\vdots \\
\mathbf{x}_{i-d}
\end{array}\right)=\mathbf{H}_{T}\left(\begin{array}{c}
\mathbf{s}_{i} \\
\mathbf{s}_{i-1} \\
\vdots \\
\mathbf{s}_{i-d-L}
\end{array}\right)+\underbrace{\left(\begin{array}{c}
\mathbf{v}_{i} \\
\mathbf{v}_{i-1} \\
\vdots \\
\mathbf{v}_{i-d}
\end{array}\right)}_{\text {Call this } \mathbf{v}_{T}}
$$


where $\mathbf{H}_{T}$ is a $(d+1) N \times(d+L+1) M$ block Toeplitz matrix defined as

$$
\mathbf{H}_{T}=\left(\begin{array}{ccccccc}
\mathbf{H}_{0} & \mathbf{H}_{1} & \ldots & \mathbf{H}_{L} & \mathbf{0} & \ldots & \mathbf{0} \\
\mathbf{0} & \mathbf{H}_{0} & \mathbf{H}_{1} & \ldots & \mathbf{H}_{L} & \mathbf{0} & \vdots \\
\vdots & & \ddots & \ddots & \ldots & \ddots & \vdots \\
\mathbf{0} & \ldots & \mathbf{0} & \mathbf{H}_{0} & \mathbf{H}_{1} & \ldots & \mathbf{H}_{L}
\end{array}\right) .
$$

One can move all of the available information including the received signals and the previous decoded symbol $\mathbf{s}_{i-\Delta-1}, \mathbf{s}_{i-\Delta-2}, \ldots, \mathbf{s}_{i-d-L}$ to the left side and obtain

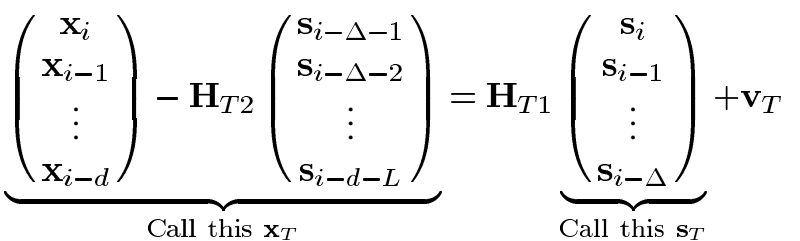

where

$$
\begin{aligned}
& \mathbf{H}_{T 1}=\mathbf{H}_{T}(:, 1:(\Delta+1) M), \text { and } \\
& \mathbf{H}_{T 2}=\mathbf{H}_{T}(:, M(\Delta+1)+1: \text { end }) .
\end{aligned}
$$

Since $\mathbf{x}_{T}$ is known, it reduces to the block channel V-BLAST system discussed in Section III except only the bottom $M$ elements of $\mathbf{s}_{T}$, namely $\mathbf{s}_{i-\Delta}$ need to be decoded instead of decoding the whole vector. This can be accomplished by using the same scheme in Fig. 1 but keeping only $M$ instead of $M(\Delta+1)$ LMMSE estimators to decode $\mathbf{s}_{i-\Delta}$. To compute the LMMSE estimators, we first compute the following variation of the $\mathrm{QR}$ decomposition:

$$
\left(\begin{array}{c}
\mathbf{H}_{T 1} \\
\sqrt{\alpha} \mathbf{I}_{(\Delta+1) M}
\end{array}\right)=\mathbf{Q}_{T}\left(\begin{array}{cc}
\times & \\
\mathbf{0}_{M \times \Delta M} & \mathbf{R}_{T}
\end{array}\right)
$$

where $\times$ denotes irrelevant terms, $\mathbf{Q}_{T}$ is unitary, and $\mathbf{R}_{T}$ is $M \times$ $M$ upper triangular with positive diagonal elements. Since only the bottom $M$ elements need to be decoded, only the bottom $M$ rows need to be upper triangular. By similar techniques used to derive (6), the LMMSE estimator of the $k$ th element of $\mathbf{s}_{i-\Delta}$ can be expressed as $\mathbf{f}_{k} \mathbf{x}_{T}^{(k)}$, where

$$
\begin{aligned}
\mathbf{x}_{T}^{(k)} & =\mathbf{x}_{T}-\sum_{m=k+1}^{M} \mathbf{H}_{T 1}(:, \Delta M+m) \hat{\mathbf{s}}_{i-\Delta}(m) \\
\mathbf{f}_{k} & =\left(\mathbf{R}_{T}(k, k)\right)^{-1}\left(\mathbf{Q}_{T}(1:(d+1) N, \Delta M+k)\right)^{\dagger} .
\end{aligned}
$$

By the same argument as for (7), the MSE of the $k$ th element can be expressed as

$$
E\left|\mathbf{s}_{i-\Delta}(k)-\mathbf{f}_{k} \mathbf{x}_{T}^{(k)}\right|^{2}=\left(\mathbf{R}_{T}(k, k)\right)^{-2} .
$$

The FIR DFE method is schematically shown in Fig. 5. The time-domain feedback block cancels the interference from the previous decoded vectors $\left\{\mathbf{s}_{k}\right\}_{k<i-\Delta}$ as shown in (26). This is the decision feedback in the time domain. The V-BLAST block performs the LMMSE estimations and cancels the interference from the decoded symbols transmitted by other antennas. This can be viewed as the decision feedback in the spatial domain.

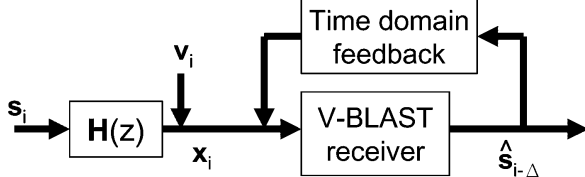

Fig. 5. FIR DFE for frequency-selective MIMO channels.

\section{B. MSE-Equalizing Precoder for the FIR DFE}

For the MIMO frequency-selective channels, we now focus on the precoder which forces the transmitted symbol streams to have identical MSEs. In Fig. 6, this MSE-equalizing precoder $\mathbf{P}_{T}$ is used in the transmitter. From (28), the MSE is related to the diagonal elements of $\mathbf{R}_{T}$. Hence, our goal is to find a unitary precoder so that the matrix $\mathbf{R}_{T}$ has identical diagonal elements. It can be obtained by the following GMD:

$$
\mathbf{R}_{T}=\mathbf{Q}_{T 1} \mathbf{R}_{T 1} \mathbf{P}_{T}^{\dagger}
$$

where $\mathbf{Q}_{T 1}$ and $\mathbf{P}_{T}$ are unitary, and $\mathbf{R}_{T 1}$ is an upper triangular matrix with

$$
\begin{aligned}
\mathbf{R}_{T 1}(k, k) & =\left|\mathbf{R}_{T}^{\dagger} \mathbf{R}_{T}\right|^{(1 / 2 M)}=\left(\prod_{i=1}^{M} \mathbf{R}_{T}(i, i)\right)^{(1 / M)} \\
\quad \text { for } k & =1,2, \ldots, M .
\end{aligned}
$$

Let the unitary matrix $\mathbf{P}_{T}$ be the precoder. The equivalent channel becomes $\mathbf{H}(z) \mathbf{P}_{T}$ and the corresponding block Toeplitz matrix defined in (25) becomes

$$
\mathbf{H}_{T} \cdot \operatorname{diag}\left\{\mathbf{P}_{T}, \mathbf{P}_{T}, \ldots, \mathbf{P}_{T}\right\} .
$$

Therefore, after precoded by $\mathbf{P}_{T}$, the submatrix of $\mathbf{H}_{T}, \mathbf{H}_{T 1}$ becomes $\mathbf{H}_{T 1} \mathbf{P}_{T d}$, where $\mathbf{P}_{T d}=\operatorname{diag}\left\{\mathbf{P}_{T}, \mathbf{P}_{T}, \ldots, \mathbf{P}_{T}\right\}$. Now we can write

$$
\left(\begin{array}{c}
\mathbf{H}_{T 1} \mathbf{P}_{T d} \\
\sqrt{\alpha} \mathbf{I}_{(\Delta+1) M}
\end{array}\right)=\left(\begin{array}{cc}
\mathbf{I} & \mathbf{0} \\
\mathbf{0} & \mathbf{P}_{T d}^{\dagger}
\end{array}\right)\left(\begin{array}{c}
\mathbf{H}_{T 1} \\
\sqrt{\alpha} \mathbf{I}_{(\Delta+1) M}
\end{array}\right) \mathbf{P}_{T d}
$$

and substitute for $\mathbf{H}_{T 1}$ from (27) with $\mathbf{R}_{T}$ as in (29). The result is

$$
\begin{aligned}
& \left(\begin{array}{c}
\mathbf{H}_{T 1} \mathbf{P}_{T d} \\
\sqrt{\alpha} \mathbf{I}_{(\Delta+1) M}
\end{array}\right)
\end{aligned}
$$

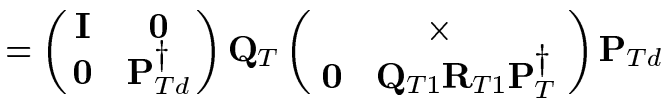

$$
\begin{aligned}
& =\underbrace{\left(\begin{array}{cc}
\mathbf{I} & \mathbf{0} \\
\mathbf{0} & \mathbf{P}_{T d}^{\dagger}
\end{array}\right) \mathbf{Q}_{T}\left(\begin{array}{cc}
\mathbf{I} & \mathbf{0} \\
\mathbf{0} & \mathbf{Q}_{T 1}
\end{array}\right)}_{\text {unitary matrix }}\left(\begin{array}{cc} 
& \times \\
\mathbf{0} & \mathbf{R}_{T 1}
\end{array}\right) .
\end{aligned}
$$

Thus, after precoding by $\mathbf{P}_{T}$, the triangular matrix in the decomposition becomes $\mathbf{R}_{T 1}$ which has identical diagonal elements obtained from the GMD in (29). The MSE becomes identical for $1 \leq k \leq M$

$$
\begin{aligned}
E\left|\mathbf{f}_{k} \mathbf{x}_{T}^{(k)}-\mathbf{s}_{i-\Delta}(k)\right|^{2} & =\left(\mathbf{R}_{T 1}(k, k)\right)^{-2} \\
& =\left(\prod_{i=1}^{M}\left(\mathbf{R}_{T}(i, i)\right)^{-2}\right)^{(1 / M)} .
\end{aligned}
$$




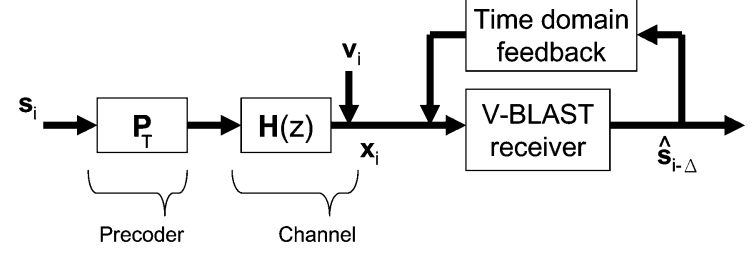

Fig. 6. Precoded FIR DFE for frequency-selective MIMO channels.

Therefore, the overall performance of the precoded V-BLAST system depends on the geometric mean of the diagonal elements of $\mathbf{R}_{T}$ which is obtained from the decomposition of $\mathbf{H}_{T 1}$ in (27).

The MSE-equalizing precoder has been derived above. However, the optimal powerloading precoder as described in Section IV-D may not be easy to compute. The powerloading precoder replaces the Toeplitz matrix $\mathbf{H}_{T}$ in (24) with $\mathbf{H}_{T} \mathbf{F}_{T}$, where $\mathbf{F}_{T}$ is a block Toeplitz matrix. The SVD technique of Section IV-D is not applicable anymore because of the restricted structure of $\mathbf{F}_{T}$.

\section{PRECODED REDUNDANT V-BLAST FOR FREQUENCY-SELECTIVE MIMO CHANNELS}

In this section, we generalize the precoded V-BLAST system for frequency-selective MIMO channels by using precoders to introduce redundancy. In the previous section, the frequency-selective MIMO channel is equalized by the FIR DFE. In the block V-BLAST systems discussed in Section III, the error propagations only exist within one block. However, the error propagations in FIR DFE system might last forever because of the time-domain feedback. This degrades the BER performance. One way to solve this is by introducing the error-control codes which correct the symbol before it is fed back. Another way is by converting frequency-selective MIMO channels into block MIMO channels by inserting redundancy, such as zero padding (ZP) or cyclic prefixing (CP). These two techniques both require inserting redundancy. In Section VII-A, simulations show that with the same amount of redundancy, the ZP- and CP-based systems have better performances than the FIR-based systems with channel coding.

In this section, we discuss precoded V-BLAST systems with $\mathrm{ZP}$ or $\mathrm{CP}$ used in the transmitter. It will be shown that the complexity of computing the LMMSE estimators and the corresponding precoders in the precoded V-BLAST systems with CP can be significantly reduced if DFT matrices are further used to block diagonalize the channel matrix.

\section{A. Precoded Zero-Padded V-BLAST for Frequency-Selective MIMO Channels}

The frequency-selective MIMO channel model is given in (23). Let $N_{p}$ zero vectors be sent after every $K$ symbol. That is, in $K+N_{p}$ symbol durations, the following is transmitted: $\left\{\mathbf{s}_{1}, \mathbf{s}_{2}, \ldots, \mathbf{s}_{K}, \mathbf{0}_{M \times 1}, \mathbf{0}_{M \times 1}, \ldots, \mathbf{0}_{M \times 1}\right\}$. In order to prevent the contamination from the previous block, one must choose $N_{p} \geq L$. The input-output relation of the system can be expressed as

$$
\begin{aligned}
& \underbrace{\left(\begin{array}{c}
\mathbf{x}_{1} \\
\mathbf{x}_{2} \\
\vdots \\
\mathbf{x}_{K+L}
\end{array}\right)}_{\text {Call this } \mathbf{x}_{p}}=\mathbf{H}_{p} \underbrace{\left(\begin{array}{c}
\mathbf{s}_{1} \\
\mathbf{s}_{2} \\
\vdots \\
\mathbf{s}_{K}
\end{array}\right)}_{\text {Call this } \mathbf{s}_{p}}+\underbrace{\left(\begin{array}{c}
\mathbf{v}_{1} \\
\mathbf{v}_{2} \\
\vdots \\
\mathbf{v}_{K+L}
\end{array}\right)}_{\text {Call this } \mathbf{v}_{p}} \\
& \text { where } \\
& \mathbf{H}_{p}=\left(\begin{array}{cccc}
\mathbf{H}_{0} & \mathbf{0} & \ldots & \mathbf{0} \\
\mathbf{H}_{1} & \mathbf{H}_{0} & \ddots & \vdots \\
\vdots & \vdots & \ddots & \mathbf{0} \\
\mathbf{H}_{L} & \vdots & \ddots & \mathbf{H}_{0} \\
\mathbf{0} & \mathbf{H}_{L} & \ddots & \vdots \\
\vdots & \ddots & \ddots & \vdots \\
\mathbf{0} & \ldots & \mathbf{0} & \mathbf{H}_{L}
\end{array}\right) .
\end{aligned}
$$

Note that as long as $N_{p} \geq L$, the input-output relation will not be affected even if we choose a larger value for $N_{p}$. This is because the extra zero padding only creates extra zeros in the end of the received block. These extra zeros contain no information about the symbols and should be discarded. The input-output relation reduces to a block MIMO channel. The V-BLAST receiver and the corresponding MSE-equalizing and power-loading precoders discussed in Sections III and IV can also be applied in this case. This system is schematically shown in Fig. 7. To compute the LMMSE estimators and the corresponding MSE-equalizing precoder, we first compute the following GMD:

$$
\left(\begin{array}{c}
\mathbf{H}_{p} \\
\sqrt{\alpha} \mathbf{I}_{K M}
\end{array}\right)=\mathbf{Q}_{p} \mathbf{R}_{p} \mathbf{P}_{p}^{\dagger}
$$

where $\mathbf{Q}_{p}$ and $\mathbf{P}_{p}$ are unitary and $\mathbf{R}_{p}$ is an upper triangular matrix with

$$
\begin{aligned}
\mathbf{R}_{p}(j, j) & =\operatorname{det}\left(\mathbf{H}_{p}^{\dagger} \mathbf{H}_{p}+\alpha \mathbf{I}_{K M}\right)^{(1 / 2 K M)} \\
& \text { for } j=1,2, \ldots, K M .
\end{aligned}
$$

By similar derivation as for (6), the LMMSE estimator of the $k$ th element of $\mathbf{s}_{p}$ can be expressed as $\mathbf{f}_{k} \mathbf{x}_{p}^{(k)}$, where

$$
\begin{aligned}
\mathbf{x}_{p}^{(k)} & =\mathbf{x}_{p}-\sum_{m=k+1}^{K M} \mathbf{H}_{p}(:, m) \mathbf{s}_{p}(m) \\
\mathbf{f}_{k} & =\left(\mathbf{R}_{p}(k, k)\right)^{-1}\left(\mathbf{Q}_{p}(1: K N, k)\right)^{\dagger} .
\end{aligned}
$$

By similar derivation as for (7), the MSE corresponding to the $k$ th symbol can be expressed as

$$
\begin{aligned}
E\left|\mathbf{s}_{p}(k)-\mathbf{f}_{k} \mathbf{x}_{p}^{(k)}\right|^{2} & =\left(\mathbf{R}_{p}(k, k)\right)^{-2} \\
& =\operatorname{det}\left(\mathbf{H}_{p}^{\dagger} \mathbf{H}_{p}+\alpha \mathbf{I}_{K M}\right)^{-(1 / M)} .
\end{aligned}
$$

By the same derivation as in Section IV-D, the symbol MSE can be further minimized to

$$
\min _{(1 / K M) \operatorname{tr}\left(\mathbf{F}_{p} \mathbf{F}_{p}^{\dagger}\right)=1} \operatorname{det}\left(\mathbf{F}_{p}^{\dagger} \mathbf{H}_{p}^{\dagger} \mathbf{H}_{p} \mathbf{F}_{p}+\alpha \mathbf{I}_{K M}\right)^{-(1 / M)}
$$




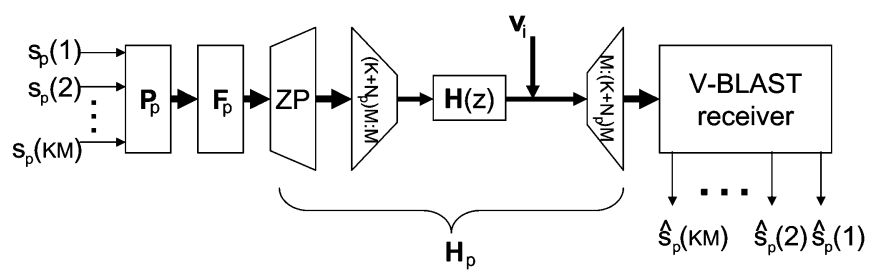

Fig. 7. Precoded ZP V-BLAST system for frequency-selective MIMO channels.

by the powerloading precoder. However, in the GMD algorithm, the SVD of the matrix has to be computed first [11]. The block size $K$ is often large in order to keep the bandwidth expansion ratio

$$
\frac{\left(K+N_{p}\right)}{K}
$$

close to unity. Therefore, the size of the matrix to be decomposed in (31) which is equal to $\left(2 K+N_{p}\right) N \times K M$ is often large. Thus, the SVD requires large amounts of computation. The use of cyclic prefix solves this problem as shown next.

\section{B. Precoded Cyclic-Prefixed V-BLAST for Frequency-Selective MIMO Channels}

The frequency-selective MIMO channel can also be converted into a block channel by using cyclic prefixing instead of ZP. In this case, the equivalent block channel matrix is block circulant. Using this property, the channel can be block diagonalized to reduce the computations for the GMD algorithm. Let $N_{p}$ be the length of the cyclic prefix sent before $K$ symbols. That is, the following symbols are transmitted in a $\left(K+N_{p}\right)$-long symbol period:

$$
\{\underbrace{\mathbf{s}_{K-N_{p}+1}, \mathbf{s}_{K-N p+2}, \ldots, \mathbf{s}_{K}}_{\mathrm{CP}}, \mathbf{s}_{0}, \mathbf{s}_{1}, \ldots, \mathbf{s}_{K}\} .
$$

At the receiver, the cyclic prefix part is discarded because it is contaminated by the response of the previous block. In order to prevent further contamination from the previous block, one must choose $N_{p} \geq L$. The input-output relation of the system can be expressed as

$$
\begin{aligned}
\underbrace{\left(\begin{array}{c}
\mathbf{x}_{1} \\
\mathbf{x}_{2} \\
\vdots \\
\mathbf{x}_{K}
\end{array}\right)}_{\text {Call this } \mathbf{x}_{p}}=\underbrace{\mathbf{H}_{c}}_{\text {Call this } \mathbf{s}_{p}} \underbrace{\left(\begin{array}{c}
\mathbf{s}_{1} \\
\mathbf{s}_{2} \\
\vdots \\
\mathbf{s}_{K}
\end{array}\right)}_{\text {Call this }}+\underbrace{\left(\begin{array}{c}
\mathbf{v}_{1} \\
\mathbf{v}_{2} \\
\vdots \\
\mathbf{v}_{K},
\end{array}\right)}_{\text {where }} \\
\mathbf{H}_{c}=\left(\begin{array}{cccccc}
\mathbf{H}_{0} & \mathbf{0} & \mathbf{0} & \mathbf{H}_{L} & \ldots & \mathbf{H}_{1} \\
\mathbf{H}_{1} & \mathbf{H}_{0} & \mathbf{0} & \mathbf{0} & \ddots & \vdots \\
\vdots & \mathbf{H}_{1} & \ddots & \ddots & \ddots & \mathbf{H}_{L} \\
\mathbf{H}_{L} & \vdots & \ddots & \ddots & & \mathbf{0} \\
\mathbf{0} & \ddots & & \ddots & \mathbf{H}_{0} & \mathbf{0} \\
\vdots & \mathbf{0} & \mathbf{H}_{L} & \ldots & \mathbf{H}_{1} & \mathbf{H}_{0}
\end{array}\right) .
\end{aligned}
$$

Note that as long as $N_{p} \geq L$, the input-output relation will not be affected even if we choose a larger value for $N_{p}$. This is because the extra cyclic prefix will be discarded at the receiver as well. The input-output relation is reduced to a block MIMO channel. The V-BLAST receiver and the corresponding optimal precoder as in Sections III and IV can be applied. This system is schematically shown in Fig. 8. By the same derivation as in Sections III and IV, the coefficients of the LMMSE estimators and the corresponding MSE-equalizing precoder can be obtained by the following GMD:

$$
\left(\begin{array}{c}
\mathbf{H}_{c} \\
\sqrt{\alpha} \mathbf{I}_{K M}
\end{array}\right)=\mathbf{Q}_{c} \mathbf{R}_{c} \mathbf{P}_{c}^{\dagger}
$$

where $\mathbf{Q}_{c}$ and $\mathbf{P}_{c}$ are unitary, and $\mathbf{R}_{c}$ is an upper triangular matrix with

$$
\begin{aligned}
\mathbf{R}_{c}(j, j) & =\left(\operatorname{det}\left(\mathbf{H}_{c}^{\dagger} \mathbf{H}_{c}+\alpha \mathbf{I}_{K M}\right)\right)^{(1 / 2 K M)} \\
\quad \text { for } k & =1,2, \ldots, K M .
\end{aligned}
$$

To reduce the computation for the SVD in the GMD algorithm, one can take advantage of the block circulant structure of the matrix $\mathbf{H}_{c}$. Because of the block circulant property, the matrix $\mathbf{H}_{c}$ can be block-diagonalized by

$$
\underbrace{\left(\mathbf{W}_{K} \otimes \mathbf{I}_{N}\right)}_{\text {Call this } \boldsymbol{\Phi}_{K}^{(N)}} \mathbf{H}_{c} \underbrace{\left(\mathbf{W}_{K} \otimes \mathbf{I}_{M}\right)^{\dagger}}_{\text {Call this } \boldsymbol{\Phi}_{K}^{(M)} \dagger}=\underbrace{\operatorname{diag}\left\{\hat{\mathbf{H}}_{1}, \hat{\mathbf{H}}_{2}, \ldots, \hat{\mathbf{H}}_{K}\right\}}_{\text {Call this } \mathbf{H}_{d}}
$$

where $\mathbf{W}_{K}$ is the $K \times K$ DFT matrix defined as

$$
\begin{aligned}
\mathbf{W}_{K}(n, m) & =\frac{1}{\sqrt{K}} e^{j(2 \pi / K)(n-1)(m-1)}, \\
\text { for } n, m & =1,2, \ldots, K, \\
\text { and } \hat{\mathbf{H}}_{k} & =\frac{1}{\sqrt{K}} \sum_{n=0}^{L} \mathbf{H}_{n} e^{-j(2 \pi / K)(k-1) n} \\
\text { for } k & =1,2, \ldots, K .
\end{aligned}
$$

By using the DFT matrices, we can block-diagonalize the matrix as

$$
\begin{aligned}
\left(\begin{array}{c}
\mathbf{H}_{c} \\
\sqrt{\alpha \mathbf{I}_{K M}}
\end{array}\right)= & \left(\begin{array}{c}
\Phi_{K}^{(N) \dagger} \mathbf{H}_{d} \Phi_{K}^{(M)} \\
\sqrt{\alpha} \mathbf{I}_{K M}
\end{array}\right) \\
= & \left(\begin{array}{cc}
\Phi_{K}^{(N) \dagger} & \mathbf{0} \\
\mathbf{0} & \mathbf{I}_{K M}
\end{array}\right)\left(\begin{array}{c}
\mathbf{H}_{d} \\
\sqrt{\alpha} \mathbf{I}_{K M}
\end{array}\right) \\
& \left.\times \begin{array}{cc}
\Phi_{K}^{(M)} & \mathbf{0} \\
\mathbf{0} & \mathbf{I}_{K M}
\end{array}\right) \\
= & \underbrace{\left(\begin{array}{cc}
\Phi_{K}^{(N) \dagger} & \mathbf{0} \\
\mathbf{0} & \mathbf{I}_{K M}
\end{array}\right) \Pi}_{\text {Unitary }} \\
& \times \mathbf{H}_{\alpha d} \underbrace{\left(\begin{array}{cc}
\left(\Phi_{K}^{(M)}\right. & \mathbf{0} \\
\mathbf{0} & \mathbf{I}_{K M}
\end{array}\right)}_{\text {Unitary }}
\end{aligned}
$$

where

$$
\begin{aligned}
& \mathbf{H}_{\alpha d}=\operatorname{diag}\left\{\mathbf{H}_{\alpha 1}, \mathbf{H}_{\alpha 2}, \ldots, \mathbf{H}_{\alpha K}\right\} \\
& \mathbf{H}_{\alpha k}=\left(\begin{array}{c}
\hat{\mathbf{H}}_{k} \\
\sqrt{\alpha} \mathbf{I}_{M}
\end{array}\right)
\end{aligned}
$$




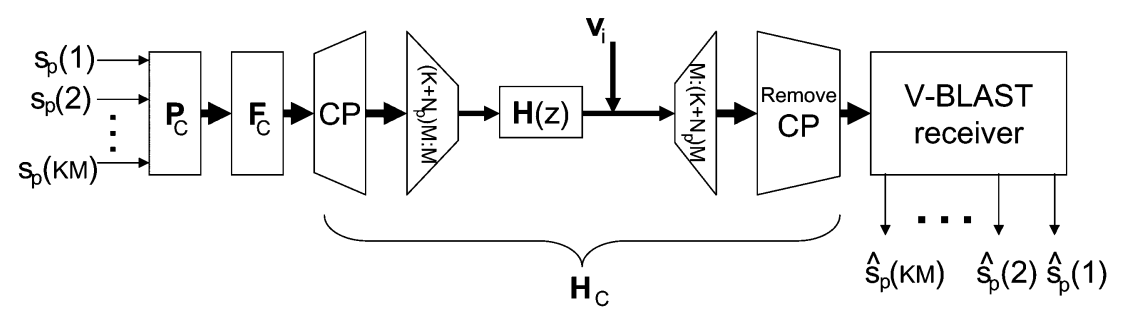

Fig. 8. Precoded CP V-BLAST system for the frequency-selective MIMO channels.

and $\boldsymbol{\Pi}$ is a permutation matrix chosen so that $\mathbf{H}_{\alpha d}$ is block diagonal. After the block diagonalization, the SVD of the block-diagonal matrix $\mathbf{H}_{\alpha d}$ can be obtained by computing the SVD of the diagonal submatrices $\mathbf{H}_{\alpha k}, k=1,2, \ldots, K$ which is much easier to compute because of the smaller size. Substituting the SVD of $\mathbf{H}_{\alpha d}$ into (32), the SVD of the original matrix is obtained. By block diagonalizing the matrix using DFT and the permutation, the complexity for the SVD in the GMD algorithm [11] can be greatly reduced. By similar derivation as for (6), the LMMSE estimator of the $k$ th element of $\mathbf{s}_{p}$ can be expressed as $\mathbf{f}_{k} \mathbf{x}_{p}^{(k)}$, where

$$
\begin{aligned}
\mathbf{x}_{p}^{(k)} & =\mathbf{x}_{p}-\sum_{m=k+1}^{K M} \mathbf{H}_{c}(:, m) \mathbf{s}_{p}(m) \\
\mathbf{f}_{k} & =\left(\mathbf{R}_{c}(k, k)\right)^{-1}\left(\mathbf{Q}_{c}(1: K N, k)\right)^{\dagger}
\end{aligned}
$$

By similar derivation as for (7), the MSE corresponding to the $k$ th symbol can be expressed as

$$
\begin{aligned}
E\left|\mathbf{s}_{p}(k)-\mathbf{f}_{k} \mathbf{x}_{p}^{(k)}\right|^{2} & =\left(\mathbf{R}_{c}(k, k)\right)^{-2} \\
& =\operatorname{det}\left(\mathbf{H}_{c}^{\dagger} \mathbf{H}_{c}+\alpha \mathbf{I}_{K M}\right)^{-(1 / M)}
\end{aligned}
$$

By the same argument as in Section IV-D, the symbol MSE can be further minimized by the power-loading precoder. The resulting MSE is

$\min _{(1 / K M) \operatorname{tr}\left(\mathbf{F}_{c} \mathbf{F}_{c}^{\dagger}\right)=1}\left(\operatorname{det}\left(\mathbf{F}_{c}^{\dagger} \mathbf{H}_{c}^{\dagger} \mathbf{H}_{c} \mathbf{F}_{c}+\alpha \mathbf{I}_{K M}\right)\right)^{-(1 / K M)}$.

To avoid the large amounts of computation spent in the SVD, we take advantage of the structure of the equivalent block channel matrix. By using $\mathrm{CP}$, the channel matrix has been converted into a block circulant matrix $\mathbf{H}_{c}$. Then, the circulant channel matrix has been further block-diagonalized by DFT and IDFT matrices. After the block diagonalization, we can use some permutations so that the corresponding matrix to be decomposed in (31) is also block diagonal. The SVD required in the GMD algorithm can thus be computed by performing the SVD on each diagonal submatrix independently. Since the sizes of the diagonal submatrices are much smaller, the complexity to compute these SVDs is small. The original SVD requires $O\left(N M^{2} K^{3}\right)$ floating point operations but now the block-diagonalized SVD requires only $O\left(N M^{2} K\right)$ [20]. By the low complexity of DFT matrices and the SVD of the diagonal submatrices, the complexity can thus be greatly reduced.

\section{Precoded OFDM V-BLAST for Frequency-Selective MIMO Channels}

If the IDFT matrices $\boldsymbol{\Phi}_{K}^{(M) \dagger}$ and the DFT matrices $\boldsymbol{\Phi}_{K}^{(N)}$ are used in the transmitter and the receiver, the system with $\mathrm{CP}$ becomes an MIMO OFDM system. Then the input-output relation of the system becomes

$$
\boldsymbol{\Phi}_{K}^{(N)} \mathbf{x}_{p}=\underbrace{\boldsymbol{\Phi}_{K}^{(N)} \mathbf{H}_{c} \boldsymbol{\Phi}_{K}^{(M) \dagger}}_{\mathbf{H}_{d}} \mathbf{s}_{p}+\underbrace{\boldsymbol{\Phi}_{K}^{(N)} \mathbf{v}_{p}}_{\text {Call this } \mathbf{v}_{p}} .
$$

It can be viewed as a block MIMO channel. Furthermore, $E \mathbf{V}_{p} \mathbf{V}_{p}^{\dagger}=\mathbf{I}_{N}$ because $\boldsymbol{\Phi}_{K}^{(N)}$ is unitary. Therefore, the precoded V-BLAST system can be obtained by the following GMD:

$$
\left(\begin{array}{c}
\mathbf{H}_{d} \\
\alpha \mathbf{I}_{K M}
\end{array}\right)=\mathbf{Q}_{d} \mathbf{R}_{d} \mathbf{P}_{d}^{\dagger}
$$

where $\mathbf{Q}_{d}$ and $\mathbf{P}_{d}$ are unitary and $\mathbf{R}_{d}$ is an upper triangular matrix with the same diagonal elements. From the second equality in (32), $\left(\begin{array}{c}\mathbf{H}_{c} \\ \alpha \mathbf{I}_{K M}\end{array}\right)$ and $\left(\begin{array}{c}\mathbf{H}_{d} \\ \alpha \mathbf{I}_{K M}\end{array}\right)$ are similar matrices. This implies that $R_{c}(k, k)=R_{d}(k, k)$ for $k=1,2, \ldots, K M$ and the precoded OFDM V-BLAST system and precoded CP V-BLAST system have the same performance. These two systems can be viewed as equivalent, because the precoded $\mathrm{CP}$ V-BLAST system has already multiplied the IDFT and DFT matrices in the precoder and the LMMSE estimators. There are no benefits implementing the DFT and IDFT matrices separately from the precoder and the LMMSE estimators. Therefore, we restrict attention to consider the precoded $\mathrm{CP}$ $\mathrm{V}$-BLAST system in this paper.

\section{Asymptotic Capacity Losslessness of Precoded CP V-blast}

In Section IV-E, we have discussed the capacity of the UCD system for block MIMO channel and mentioned that it is identical to the capacity of the MIMO channel. In this section, we derive the capacity of the precoded CP V-BLAST system based on the results in Section IV-E. Substituting the equivalent block channel $\mathbf{H}_{c}$ into (22), the capacity of the precoded OFDM V-BLAST system per channel use can be expressed as

$$
\begin{aligned}
C_{c p}= & \frac{1}{K+N_{p}} \\
& \times \max _{(1 / K M) \operatorname{tr}\left(\mathbf{R}_{s}\right)=(1 / \alpha)} \log \operatorname{det}\left(\mathbf{H}_{c} \mathbf{R}_{s}^{\dagger} \mathbf{H}_{c}^{\dagger}+\mathbf{I}_{K N}\right) \\
= & \frac{1}{K+N_{p}} \\
& \times \max _{(1 / K M) \operatorname{tr}\left(\mathbf{R}_{s}\right)=(1 / \alpha)} \log \operatorname{det}\left(\mathbf{H}_{d} \mathbf{R}_{s}^{\dagger} \mathbf{H}_{d}^{\dagger}+\mathbf{I}_{K N}\right) .
\end{aligned}
$$


Since $\mathbf{H}_{d}$ is block diagonal, the solution of $\mathbf{R}_{s}$ is also block diagonal because the off block-diagonal elements can only decrease the determinant. Letting $\mathbf{R}_{s}=\operatorname{diag}\left\{\mathbf{R}_{1}, \mathbf{R}_{2}, \ldots, \mathbf{R}_{K}\right\}$, the capacity can be further expressed as

$$
\begin{gathered}
C_{c p}=\max _{\mathbf{R}_{i}} \sum_{i=1}^{K} \log \operatorname{det}\left(\mathbf{H}_{i} \mathbf{R}_{i} \mathbf{H}_{i}^{\dagger}+\mathbf{I}_{N}\right) \frac{1}{K+N_{p}} \\
\text { subject to } \frac{1}{K M} \sum_{i=1}^{K} \operatorname{tr}\left(\mathbf{R}_{i}\right)=\frac{1}{\alpha} .
\end{gathered}
$$

$\left\{\mathbf{H}_{i}\right\}$ are the DFT coefficients of the channel $\mathbf{H}(z)$. They can be expressed as $\mathbf{H}_{i}=\mathbf{H}\left(e^{j((2 \pi / K)(i-1))}\right)$ for $i=1,2, \ldots, K$. Substituting this into the above equation and taking $K \rightarrow \infty$, the capacity becomes

$$
\begin{aligned}
& \lim _{K \rightarrow \infty} C_{c p} \\
&=\max _{\mathbf{R}(f)} \int_{0}^{1} \log \operatorname{det}\left(\mathbf{H}\left(e^{j 2 \pi f}\right) \mathbf{R}(f) \mathbf{H}^{\dagger}\left(e^{j 2 \pi f}\right)+\mathbf{I}_{N}\right) d f \\
& \text { subject to }\left(\frac{1}{M}\right) \int_{0}^{1} \operatorname{tr}(\mathbf{R}(f)) d f=\left(\frac{1}{\alpha}\right) .
\end{aligned}
$$

This is equal to the capacity of the frequency-selective MIMO channel [18], [19]. That is, $\lim _{K \rightarrow \infty} C_{c p}=C_{i s i \text {-mimo, where }}$ $C_{i s i-m i m o}$ is the capacity of the frequency-selective MIMO channel. We have shown that the precoded CP V-BLAST system is asymptotically capacity lossless. That is, in the precoded CP V-BLAST system, the sum of the capacity of the equalized SISO channels approaches the capacity of the frequency-selective MIMO channel when the DFT size $K$ is increased.

\section{NUMERICAL EXAMPLES}

In this section, the BER performances of the MIMO systems for the frequency-selective channels are compared under different SNR. The SNR is $1 / \alpha$ as defined in (1). For the systems with cyclic prefixing, the SNR is defined as $\left(\left(K+N_{p}\right) / K\right) / \alpha$ in order to include the power spent by the cyclic prefix. The frequency-selective channel matrix used in the simulations is $2 \times 2$ with order $L=3$. The real and imaginary parts of the channel coefficients are generated as i.i.d. Gaussian random variables with zero mean and variance 0.5 . The real and imaginary parts of the channel noise are also generated as i.i.d. Gaussian random variables with zero mean and variance 0.5 . Therefore, the complex Gaussian random variable has a unit variance as described in the beginning of Section III. We assume the transmitter and receiver have perfect channel information and the statistics of the channel noise. The simulation is performed by averaging among many different channel and noise realizations.

\section{A. Comparison Based on Precoders}

Fig. 9 shows the BER performances of the FIR V-BLAST system described in Section V, the redundant V-BLAST systems described in Section VI, and their precoded versions, including the MSE-equalizing precoder and the powerloading precoder. The following eight systems are compared.

1) FIR-V-BLAST. This system uses FIR MMSE DFE described in Section V with an FIR order $d=2$ and decision

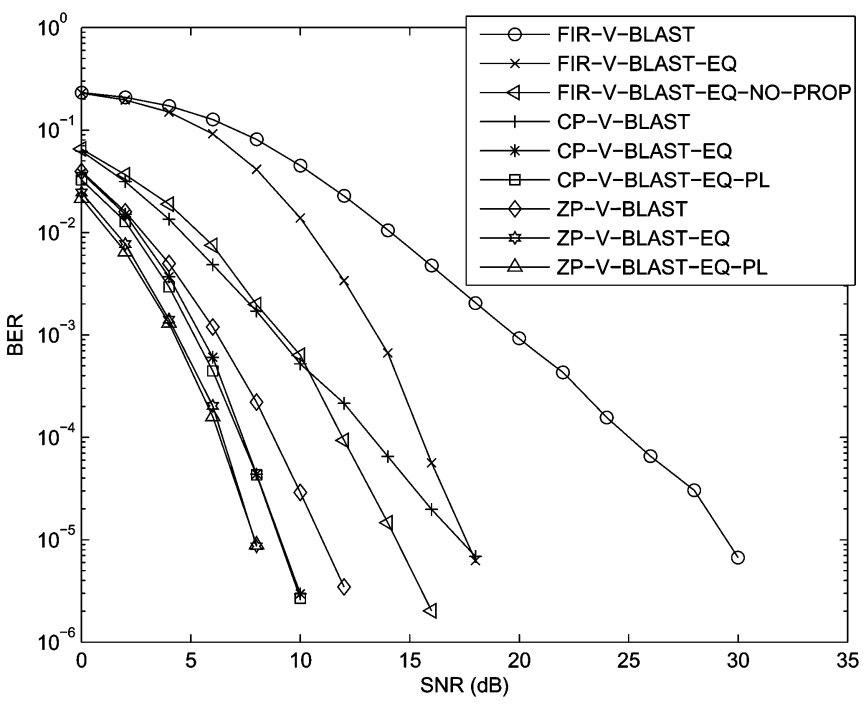

Fig. 9. Comparison of the BER performances of systems with different precoding methods.

delay $\Delta=1$. We select this $\Delta$ by manually testing the position of the decision delay. Since the channel order $L$ is 3 and the FIR order $d$ is 2, it is reasonable to choose $\Delta=1$ in order to capture most of the symbol energy into the FIR filter. This system was proposed in [12] and [13].

2) FIR-V-BLAST-EQ. This system is the FIR-V-BLAST system with the MSE-equalizing precoder.

3) FIR-V-BLAST-EQ-NO-PROP. The same as FIR-VBLAST-EQ. However, the correct symbols are fedback so that there is no error propagation. This system is not realistic practically. It is only used as a theoretical bound.

4) $C P-V-B L A S T$. This system uses cyclic prefix (CP) between blocks with length $N_{p}=3$ and block size $K=16$. The block V-BLAST is used at the receiver to decode the symbols as described in Section VI.

5) $C P-V$-BLAST-EQ. This system is the CP-V-BLAST system with the MSE-equalizing precoder.

6) $C P$-V-BLAST-EQ-PL. This system is the CP-V-BLAST system with both the MSE-equalizing precoder and the power-loading precoder.

7) $Z P$-V-BLAST. This system uses zero padding (ZP) between blocks with length $N_{p}=3$ and block size $K=16$. The block V-BLAST system is used at the receiver to decode the symbols as described in Section VI.

8) ZP-V-BLAST-EQ. This system is the ZP-V-BLAST system with the MSE-equalizing precoder.

9) ZP-V-BLAST-EQ-PL. This system is the ZP-V-BLAST system with both the MSE-equalizing precoder and the power-loading precoder.

All eight systems use QPSK symbols.

Because of the introduction of redundancy, the bit rate is reduced to $K /\left(K+N_{p}\right)$ times the bit rate of the system without redundancy. In our example, the bit rate in the CP and ZP systems is $16 / 19$ times the bit rate of the system without redundancy. The performances of the FIR-based systems (without redundancy) are not as good as the CP and ZP systems. This is because they suffer from endless error propagations. 
A natural question that arises here is whether the redundancy introduced by the $\mathrm{CP}$ and $\mathrm{ZP}$ systems can be replaced with channel coding in order to obtain the same or better performance. We now address this question.

The error propagations can be reduced by introducing the channel coding. To compare them in a fair manner, one can modify the FIR-based system by introducing channel coding with the same amount of redundancy as was used in the CPand ZP-based systems. In our experiment, rate 4/5 maximum free-distance convolutional code was applied to the FIR systems because $4 / 5$ is very close to $16 / 19$. The free distance $d_{\text {free }}$ of such a code is 3 [24]. The corresponding coding gain can be expressed as [24]

$$
\gamma=10 \log _{10}\left(d_{\text {free }}\right) \approx 4.77 \mathrm{~dB} .
$$

In Fig. 9, the difference between CP-V-BLAST-EQ and FIR-VBLAST-EQ-NO-PROP is about $6 \mathrm{~dB}$ which is greater than the coding gain of $4.77 \mathrm{~dB}$. Therefore, even without error propagations, the FIR-V-BLAST-EQ system with the rate $4 / 5$ channel code still has a worse performance than the CP-V-BLAST-EQ system. This shows that at least in these examples, with the same amount of redundancy, the CP- and ZP-based precoded systems have better performances than the FIR-based precoded systems with channel coding.

The CP-based systems send cyclic prefixes in the transmitter. They consume more power than zero padding. Thus, the performances of the CP-based systems are slightly worse than that of the ZP-based systems. The MSE-equalizing precoder improves all of the three types of systems considerably. However, there are only slight improvements when the power-loading precoders are further used. This suggests that the power-loading precoder can be ignored when implementing the precoded V-BLAST systems. The theoretical reason for this is not clear at this time. Our conjecture is that bit loading and the MSE-equalizing precoder have already exploited the eigenmode variations in the channel.

\section{B. Comparison Based on the Bit-Loading Methods}

To compare the systems which have channel information available at the transmitter, we first compare some systems with their optimal bit-loading versions. Fig. 10 shows four different MIMO systems for frequency-selective channels and their optimal bitloaded version. The following eight systems are compared.

1) OFDM-LE. The frequency-selective MIMO channel is converted into $K$ parallel block channels by OFDM with the DFT size $K=16$ and the CP length $N_{p}=3$. Each block channel matrix is equalized by an MMSE linear equalizer separately. QPSK symbols are transmitted.

2) $O F D M-L E-B L$. This system is the OFDM-LE system with optimal bit loading while fixing the bit-transmission rate.

3) OFDM-V-BLAST. The frequency-selective MIMO channel is converted into $K$ parallel block channels by OFDM with the DFT size $K=16$ and the CP length $N_{p}=3$. Each block channel is equalized by the V-BLAST decoder separately. QPSK symbols are transmitted.

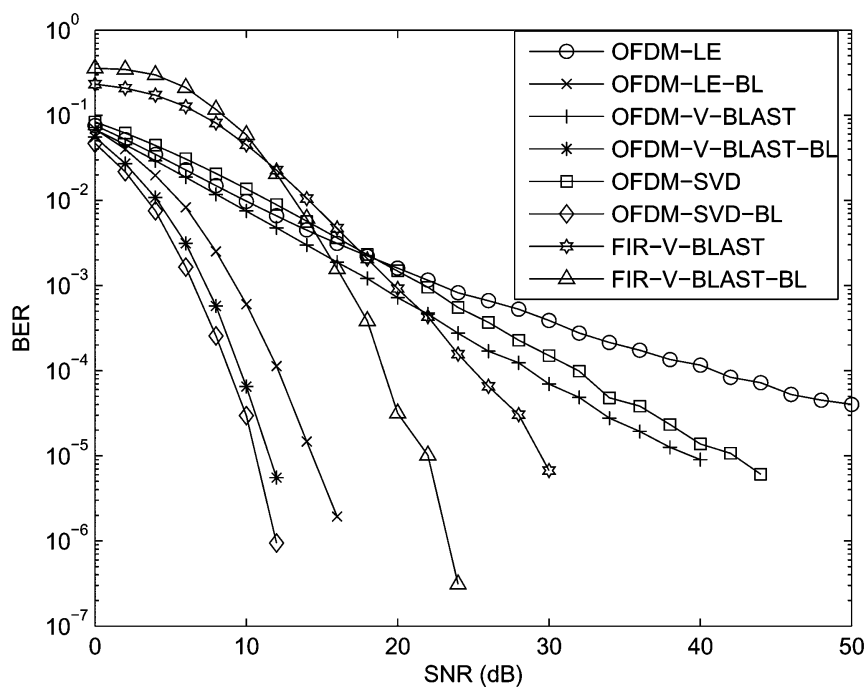

Fig. 10. Comparison of the BER performances of systems with different bitloading methods.

4) $O F D M-V-B L A S T-B L$. This system is the OFDM-V-BLAST system with optimal bit loading while fixing the bit transmission rate.

5) $O F D M-S V D$. The frequency-selective MIMO channel is converted into $K$ parallel block channels by OFDM with the DFT size $K=16$ and the CP length $N_{p}=3$. Each block channel is further converted into $M$ scalar channels by SVD. The scalar MMSE equalizers are used in all of the $K M$ scalar channels separately.

6) OFDM-SVD-BL. This system is the OFDM-SVD system with optimal bit loading while fixing the bit transmission rate.

7) FIR-V-BLAST. Similar to Section VII-A (1).

8) FIR-V-BLAST-BL. This system is the FIR-V-BLAST system with optimal bit loading while fixing the bit transmission rate.

Bit loading improves the BER performances a lot especially in the SVD-based systems. The OFDM-SVD-BL system has the best BER performance among all optimal bit-loaded systems. Among the systems without channel information used in the transmitter, the FIR-V-BLAST has the best performance. However, it has a relatively small improvement when bit loading is used. The FIR-V-BLAST-BL system has the worst BER performance among the systems with bit loading. Among the OFDMbased systems, the SVD-based systems are generally better than others.

\section{Comparison of the Systems With Channel Information Used in the Transmitter}

We now compare all of the systems using the channel information in the transmitters. These systems employ the best strategies at both the transmitter and receiver. Fig. 11 shows the BER performances of the following seven systems.

1) $O F D M-S V D-B L-P L$. This system is the OFDM-SVD-BL system in Section VII-B (6) with the power-loading precoder. This system was proposed in [16].

2) $O F D M-L E-B L$. Same as Section VII-B (2).

3) $O F D M-V-B L A S T-B L$. Same as Section VII-B (5). 


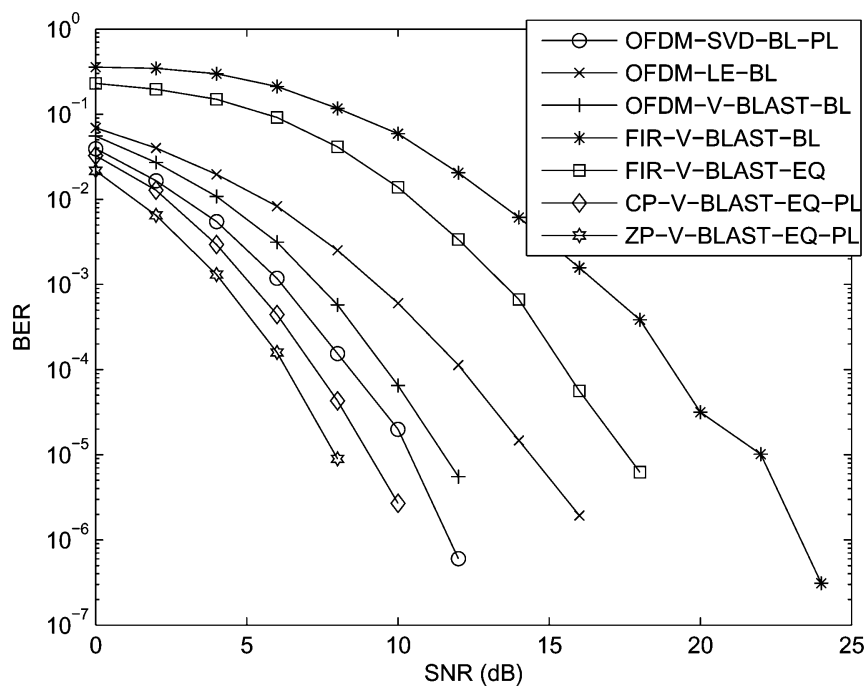

Fig. 11. Comparison of the BER performances of systems with channel information used in the transmitter.

4) FIR-V-BLAST-BL. Same as Section VII-B (8).

5) FIR-V-BLAST-EQ. Same as Section VII-A (2).

6) $C P-V-B L A S T-E Q-P L$. Same as Section VII-A (6).

7) $Z P-V-B L A S T-E Q-P L$. Same as Section VII-A (8).

The ZP-V-BLAST-EQ-PL system has the best BER performance among all of these systems. The CP-V-BLAST-EQ-PL system has the second best BER performance because it sends $\mathrm{CP}$ which consumes more power than ZP. This difference will be small when the large discrete Fourier transform (DFT) size $K$ is used. Also, the CP-based system has the fast algorithm for computing the MSE-equalizing precoder as described in Section VI-B. From the comparison in Section VII-A, we know that the gain from the power-loading precoder is slight. Therefore, we believe that the CP-V-BLAST-EQ system is a good candidate for the MIMO transceiver for frequency-selective channels when the channel information is available at the transmitter. The OFDM-SVD-BL-PL has the best performance among all of the bit-loading-based systems. However, it uses about $1 \mathrm{~dB}$ more energy than the CP-V-BLAST-EQ-PL system when transmitting symbols with a BER of less than $10^{-5}$. This gain comes from the fact that the MSE-equalizing precoder has better BER performance than the bit-loading method as we pointed out in Section IV-B.

\section{Comparison of the Systems With Inaccurate Channel Information Used in the Transmitter and the Receiver}

In the previous examples, we assume that the channel state information (CSI) is perfectly known at the transmitter and the receiver. However, the channel information can only be estimated in the receiver and fed back to the transmitter. There always exists a certain amount of estimation error in the CSI. For time-varying channels, the estimation error can be even larger because of the delay caused by the feedback of the CSI. By the time the CSI arrives at the transmitter, the channel might have

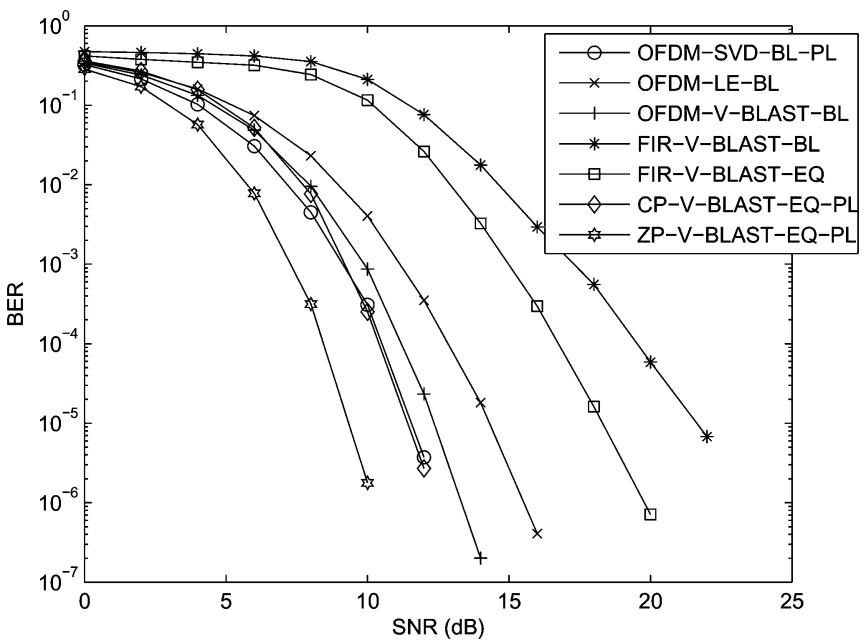

Fig. 12. Comparison of the BER performances with inaccurate channel information.

changed. Fig. 12 shows a BER comparison of the seven systems compared in Section VII-C with inaccurate channel information used in both the transmitter and the receiver. The inaccurate channel matrix used in these transceivers is modeled by

$$
\mathbf{H}_{\Delta}(z)=\sum_{k=0}^{L}\left(\mathbf{H}_{k}+\sqrt{\alpha} \boldsymbol{\Delta}_{k}\right) z^{-k}
$$

where $\mathbf{H}_{k}$ is the impulse response defined in (23), $\alpha$ is the noise-to-signal ratio defined in (1), and the real part and imaginary part of $\boldsymbol{\Delta}_{k}(i, j)$ are i.i.d. Gaussian random variables with zero mean and variance 0.5 for all $i, j$, and $k$. Comparing Fig. 12 to Fig. 11, we see that both the OFDM-SVD-BL-PL system and the OFDM-V-BLAST-BL system have less performance degradation than the CP-V-BLAST-EQ-PL system. This shows that the transceivers with the MSE-equalizing precoder are more sensitive to inaccurate CSI than the transceivers with bit loading. However, for the FIR-based systems, the difference in sensitivity is slight. The CP-based system is more sensitive to inaccurate CSI than the ZP-based system.

\section{CONCLUSION}

We have extended the UCD system proposed in [4] and [6] to the case of frequency-selective MIMO channels by the FIR and the redundant types of transceivers. These proposed transceivers convert the frequency-selective MIMO channels into multiple identical parallel scalar channels. Simple constellations, such as QPSK, can be used in these systems. Examples of these systems and the existing systems based on optimal bit loading are compared. Among these proposed systems, the CP-based V-BLAST system with the MSE-equalizing precoder described in Section VI-B has a fast algorithm for computing the MSE-equalizing precoder and the numerical simulations also show that this system has very good performance compared to the existing SVD-based system with optimal bit loading. We believe that the CP-based precoded V-BLAST system is a good candidate for the transceiver for MIMO frequency-selective channels when the channel information is available at the transmitter. 


\section{REFERENCES}

[1] B. Hassibi, "An efficient square-root algorithm for BLAST," Proc. ICCASP, vol. 2, pp. II737-II740, Jun. 1999.

[2] Y. Jiang and J. Li, "Uniform channel decomposition for MIMO communications," in Proc. 38th IEEE Asilomar Conf. Signals, Systems, and Computers, Nov. 2004, pp. 856-860.

[3] Y. Jiang, J. Li, and W. W. Hager, "Joint transceiver design for MIMO communications using geometric mean decomposition," IEEE Trans. Signal Process., vol. 53, no. 10, pp. 3791-3803, Oct. 2005.

[4] —- "Uniform channel decomposition for MIMO communications," IEEE Trans. Signal Process., vol. 53, no. 11, pp. 4283-4294, Nov. 2005.

[5] L. Yang, J. Li, and Y. Jiang, "Capacity-approacing transeiver design for asymmetric UWB links," presented at the 39th IEEE Asilomar Conf. on Signals, Systems, and Computers, Nov. 2005

[6] F. Xu, T. N. Davidson, J. K. Zhang, S. S. Chan, and K. M. Wong, "Design of block transceivers with decision feedback detection," in IEEE Trans. Signal Process., Mar. 2006, vol. 54, no. 3, pp. 965-978.

[7] — - "Design of block transceivers with MMSE decision feedback detection," Proc. ICASSP, pp. 1109-1112, Mar. 2005.

[8] J. K. Zhang, A. Kavcic, and K. M. Wong, "Equal-diagonal QR decomposition and its application to precoder design for successive-cancellation detection," IEEE Trans. Inf. Theory, vol. 51, no. 1, pp. 154-172, Jan. 2005

[9] J. K. Zhang, T. N. Davidson, and K. M. Wong, "Uniform decomposition of mutual information using MMSE decision feedback detection," Proc. ISIT, pp. 714-718, Sep. 2005.

[10] P. W. Wolniansky, G. J. Foschini, G. D. Golden, and R. A. Valenzela, "V-BLAST: An architecture for realizing very high data rates over the rich-scattering channel," in Proc. Int. Symp. Signals, Systems and Electronics, 1998, pp. 295-300.

[11] Y. Jiang, W. W. Hager, and J. Li, "The geometric mean decomposition and generalized triangular decomposition," presented at the SIAM Annu. Meeting, Portland, OR, Jul. 16, 2004.

[12] N. Al-Dhahir and A. H. Sayed, "The finite-length multi-input multioutput MMSE-DFE," IEEE Trans. Signal Process., vol. 48, no. 10, pp. 2921-2936, Oct. 2000.

[13] A. Voulgarelis, M. Joham, and W. Utschick, "Space-time equalization based on V-BLAST and DFE for frequency selective MIMO channels," Proc. ICASSP, vol. 4, pp. IV-381, Apr. 2003.

[14] G. L. Stuber, J. R. Barry, S. W. McLaughlin, S. W. Ye Li, M. A. Ingram, and T. G. Pratt, "Broadband MIMO-OFDM wireless communications," Proc. IEEE, vol. 92, no. 2, pp. 271-294, Feb. 2004.

[15] D. Palomar, J. Cioffi, and M. Lagunas, "Joint Tx-Rx beamforming design for multicarrier MIMO channels: A unified framework for convex optimization," IEEE Trans. Signal Process., vol. 51, no. 9, pp. 2381-2401, Sep. 2003.

[16] D. Palomar and S. Barbarossa, "Designing MIMO communication systems: Constellation choice and linear transceiver design," IEEE Trans. Signal Process., vol. 53, no. 10, pt. 1, pp. 3804-3818, Oct. 2005.

[17] D. N. C. Tse, P. Viswanath, and L. Zheng, "Diversity-multiplexing tradeoff in multiple-access channels," IEEE Trans. Inf. Theory., vol. 50, no. 9, pp. 1859-1874, Sep. 2004.

[18] E. Telatar, "Capacity of multi-antenna Gaussian channels," Eur. Trans. Telecommun., vol. 10, no. 6, pp. 585-595, Nov. 1999.

[19] T. M. Cover and J. A. Thomas, Elements of Information Theory. New York: Wiley, 1991.

[20] G. H. Golub and C. F. Van Loan, Matrix Computations. Baltimore, MD: Johns Hopkins Univ., 1996.

[21] J. R. Schott, Matrix Analysis for Statistics. New York: Wiley, 1996.

[22] R. A. Horn and C. R. Johnson, Matrix Analysis. Cambridge, U.K.: Cambridge University Press, 1985.

[23] S. Haykin, Communication Systems. New York: Wiley, 2001.

[24] J. G. Proakis, Digital Communicatins. New York: McGraw-Hill, 2001.

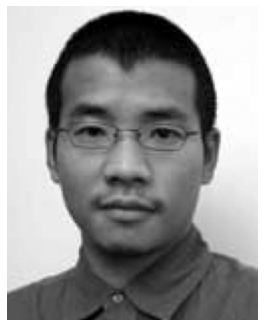

Chun-yang Chen (S'05) was born in Taipei, Taiwan, R.O.C., on November 22, 1977. He received the B.S. and M.S. degrees in electrical engineering and communication engineering from National Taiwan University (NTU), Taipei, in 2000 and 2002, respectively, and is currently pursuing the Ph.D. degree in electrical engineering in the field of digital signal processing at California Institute of Technology, Pasadena.

His interests include signal processing in MIMO communications, ultra-wideband communications, and radar applications.

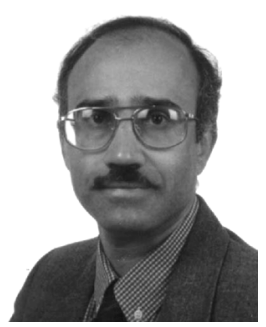

P. P. Vaidyanathan (S'80-M'83-SM'88-F'91) was born in Calcutta, India, on October 16, 1954. He received the B.Sc. (Hons.) degree in physics and the B.Tech. and M.Tech. degrees in radiophysics and electronics from the University of Calcutta, Calcutta, India, in 1974, 1977, and 1979, respectively, and the $\mathrm{Ph} . \mathrm{D}$. degree in electrical and computer engineering from the University of California at Santa Barbara in 1982.

He was a Postdoctoral Fellow at the University of California at Santa Barbara from 1982 to 1983. In 1983, he joined the Electrical Engineering Department of the California Institute of Technology, Pasadena, as an Assistant Professor, and since 1993 has been Professor of Electrical Engineering. His main research interests are digital signal processing, multirate systems, wavelet transforms, and signal processing for digital communications.

Dr. Vaidyanathan served as Vice-Chairman of the Technical Program committee for the 1983 IEEE International symposium on Circuits and Systems, and as the Technical Program Chairman for the 1992 IEEE International Symposium on Circuits and Systems. He was an Associate Editor for the IEEE TRANSACTIONS ON CIRCUITS AND SYSTEMS from 1985 to 1987, and is currently an Associate Editor for IEEE SIGNAL PROCESSING LETTERS, and a Consulting Editor for the journal Applied and Computational Harmonic Analysis. He has been a Guest Editor in 1998 for a special issues of the IEEE TRANSACTIONS ON SigNAL PROCESSING and the IEEE TRANSACTIONS ON CiRCUITS AND SYSTEMS II, on the topics of filter banks, wavelets, and subband coders. He has authored a number of papers in IEEE journals, and is the author of the book Multirate Systems and Filter Banks. He has written several chapters for various signal processing handbooks. He was a recepient of the award for excellence in teaching at the California Institute of Technology for the years 1983-1984, 1992-1993, and 1993-1994. He also received the National Science Foundation's Presidential Young Investigator Award in 1986. In 1989, he received the IEEE ASSP Senior Award for his paper on multirate perfect-reconstruction filter banks. In 1990, he was recepient of the S. K. Mitra Memorial Award from the Institute of Electronics and Telecommuncations Engineers, India, for his joint paper in the IETE Journal. He was also the coauthor of a paper on linear-phase perfect reconstruction filter banks in the IEEE TRANSACTIONS ON SIGNAL PROCESSING, for which the first author (T. Nguyen) received the Young Outstanding Author Award in 1993. He received the 1995 F. E. Terman Award of the American Society for Engineering Education, sponsored by Hewlett Packard Co., for his contributions to engineering education, especially the book Multirate Systems and Filter Banks (Prentice-Hall, 1993). He has given several plenary talks including at the SAMPTA'01, EUSIPCO'98, SPCOM'95, and ASILOMAR' 88 Conferences on signal processing. He has been chosen a Distinguished Lecturer for the IEEE Signal Processing Society for the year 1996-1997. In 1999, he was chosen to receive the IEEE Circuits and Systems Society's Golden Jubilee Medal. He is a recipient of the IEEE Signal Processing Society's Technical Achievement Award for 2002. 